\title{
Gene-environment interactions characterized by single embryo
} transcriptomics

3 Alfire Sidik ${ }^{1}$, Groves B. Dixon ${ }^{1}$, Hannah G. Kirby ${ }^{1}$, and Johann K. Eberhart ${ }^{1 *}$

$5{ }^{1}$ Department of Molecular Biosciences, Institute for Cellular and Molecular Biology,

6 Institute for Neuroscience, Waggoner Center for Alcohol and Addiction Research,

7 University of Texas, Austin, TX 78712, USA

8 *Correspondence to: Johann K. Eberhart, PAT 522, The University of Texas at Austin,

9 Molecular Biosciences, College of Natural Sciences, 2401 Speedway, Austin, TX, 78712.

10 Phone: 512-232-8340.

11

12

13

14

15

16

17

18

19

20

21

22

23 


\section{AbSTRACT}

Gene-environment interactions are likely to underlie most human birth defects. The most common environmental contributor to birth defects is likely prenatal alcohol exposure. Fetal Alcohol Spectrum Disorders (FASD) describes the full range of defects

28 that result from prenatal alcohol exposure. Gene-ethanol interactions underlie

29 susceptibility to FASD but we lack a mechanistic understanding of these interactions. Here,

30 we leverage the genetic tractability of zebrafish to address this problem. We first show that 31 vangl2, a member of the Wnt/planar cell polarity (Wnt/PCP) pathway that mediates

32 convergent extension movements, strongly interacts with ethanol during late blastula and 33 early gastrula stages. Embryos mutant or heterozygous for vangl2 are sensitized to ethanol34 induced midfacial hypoplasia. We performed single-embryo RNA-Seq during early 35 embryonic stages, to assess individual variation to the transcriptional response to ethanol 36 and determine the mechanism of the vangl2-ethanol interaction. To identify the pathway(s) 37 that are disrupted by ethanol we used these global changes in gene expression to identify 38 small molecules that mimic the effects of ethanol via the Library of Integrated Network39 based Cellular Signatures (LINCS L1000) dataset. Surprisingly, this dataset predicted that 40 the Sonic Hedgehog (Shh) pathway inhibitor, cyclopamine, would mimic the effects of 41 ethanol, despite the fact that ethanol did not alter the expression levels of direct targets of

42 Shh signaling. Indeed, we found that ethanol and cyclopamine strongly interact to disrupt 43 midfacial development. Collectively, these results suggest that the midfacial defects in 44 ethanol-exposed vangl2 mutants are due to an indirect interaction between ethanol and the 45 Shh pathway. Vangl2 functions as part of a signaling pathway that regulates coordinated 46 cell movements during midfacial development. Consistent with an indirect model, a critical

47 source of Shh signaling that separates the developing eye field into bilateral eyes, allowing 48 the expansion of the midface, becomes mispositioned in ethanol-exposed vangl2 mutants. 
49 We demonstrate that ethanol also interacts with another Wnt/PCP pathway member, gpc4,

50 and a chemical inhibitor, blebbistatin. By characterizing membrane protrusions, we

51 demonstrate that ethanol synergistically interacts with the loss of vangl2 to disrupt cell

52 polarity required for convergent extension movements. Collectively, our results shed light

53 on the mechanism by which the most common teratogen can disrupt development.

\section{INTRODUCTION}

Birth defects manifest as structural or functional malformations at birth and are the

56 leading cause of infant mortality in the United States [1]. Although the multifactorial

57 etiologies of birth defects are not well understood, many are thought to derive from a

58 complex interplay between genetic and environmental factors. The adverse effects of

59 teratogens, or environmental agents that cause irreversible developmental defects, were

60 first recognized in the 1950s and 1960s [2]. It was then that investigators began to observe

61 developmental anomalies in infants exposed to methylmercury and thalidomide in utero

$62[3,4]$. Since then, other environmental teratogens have come to light, including but not

63 limited to, pollutants, pharmaceuticals, and chemicals.

Prenatal alcohol exposure (PAE) is the most common cause of birth defects, with

65 the prevalence of fetal alcohol spectrum disorders (FASD) in the US is as high as 2-5\%

66 [5]. Fetal alcohol syndrome (FAS) is the most severe outcome following PAE and is

67 characterized by midfacial hypoplasia, as well as growth and neural deficits [6]. While

68 PAE is required for the development of FASD, the teratogenic effects of ethanol are

69 modulated by genetics [7, 8]. For instance, monozygotic twins were $100 \%$ concordant for

70 FAS, whereas dizygotic twins were only $63 \%$ concordant $[8,9]$. Furthermore, animal

71 models of FAS show strain-specific differences after controlling for environmental

72 variables such as dose and timing $[10,11]$. Despite this, the genetic factors that protect or 
73 predispose an individual to FASD are poorly understood. Moreover, we still lack a basic

74 understanding of the mechanism of ethanol teratogenesis. Zebrafish are well suited to 75 address this problem due to their genetic tractability and ease of embryological 76 manipulation [12].

77 In a screen to identify genetic modifiers of ethanol teratogenicity, vang-like 2 78 (vangl2), a member of the non-canonical Wnt/PCP pathway, emerged as an ethanol 79 sensitive locus [13]. In zebrafish, mutations in the vangl2 locus disrupt convergent 80 extension movements during gastrulation, resulting in a shortened, broadened, body axis 81 [14]. These mutants infrequently present with cyclopia, fusion of the bilateral eyes, but the 82 phenotypic expressivity of this trait varies with factors such as temperature and genetic 83 background [15]. In the screen, all untreated vangl2 mutants displayed proper separation 84 of the eyes and craniofacial skeletal elements were intact [13]. Upon exposure to a 85 subteratogenic dose of ethanol, vangl2 mutants were fully penetrant for cyclopia and 86 displayed profound defects to the midfacial skeleton [13]. Ethanol-treated vangl2 87 heterozygotes were largely indistinguishable from their wild-type siblings, with the 88 exception of a single synophthalmic ethanol-treated heterozygote, providing evidence for 89 latent haploinsufficiency. Together, these data suggest a synergistic interaction between a $90 \quad$ mutation in vangl2 and ethanol.

91 We know vangl2 plays a critical role in mediating convergent extension movements

92 as evidenced by their body axis defect, however because the early effects of ethanol 93 exposure remain poorly defined, the precise mechanism of the vangl2-ethanol interaction 94 remain elusive. To better understand how ethanol interacts with loss of vangl2 to alter 95 phenotypic outcomes, we took an unbiased approach to assess the transcriptional response 96 to ethanol. we performed single embryo RNA-sequencing (RNA-seq) on control 
97 (untreated) and ethanol-treated wild-type embryos in a time-course spanning gastrulation

98 and early segmentation stages in zebrafish.

99 Bioinformatic and functional analyses indicated that midfacial defects in ethanol-

100 exposed vangl2 mutants was due to an indirect interaction between ethanol and the Shh

101 pathway. While there was no alteration in the level of expression of direct Shh targets, a

102 critical source of Shh signaling that separates the developing eye field into bilateral eyes

103 becomes mispositioned in ethanol-exposed vangl2 mutants. We demonstrate that ethanol

104 and loss of vangl2, synergistically interact to disrupt polarized cellular protrusions required

105 for proper convergent extension movements.

\section{RESULTS}

\section{Early embryogenesis is the sensitive time window for vangl2 mutants}

108 To determine the critical time window of ethanol sensitivity for the vangl2 mutants, 109 we first initiated ethanol treatment at various stages comprising late blastula to early 110 gastrula for 24 hours. The inner lens-to-lens width was used as a measure of synophthalmia 111 and we calculated the occurrence of cyclopia to characterize ethanol-induced teratogenesis.

112 In control conditions, the spacing of the eyes in vangl2 mutants mirrored that of wild-type 113 zebrafish. Ethanol-exposed vangl2 mutants exhibited midline defects ranging in severity

114 from synophthalmia to cyclopia across all time points examined, but these malformations 115 were fully penetrant for cyclopia (100\% fused; $n=5 / 5)$ when ethanol was applied at shield 116 stage (6 hours post fertilization, hpf) at the onset of gastrulation (Fig 1A-B). Interestingly, 117 heterozygotes only displayed cyclopia when ethanol was applied at high stage (3.3 hpf) 118 (22\% fused; $n=4 / 18)$, a time when treating wild-type embryos with higher concentrations 119 of ethanol causes similar defects (Fig 1B) [16]. Thus, heterozygotes and homozygotes are 120 divergent in their time window of greatest ethanol sensitivity. This may be due to a 
121 compensatory genetic mechanism in vangl2 heterozygotes because zygotic gene

122 expression initiates after high stage (4 hpf) in zebrafish.

\section{The effect of ethanol on the early zebrafish transcriptome is subtle relative to} 124 developmental time

125 To determine if ethanol caused transcriptional changes that could underlie the 126 interaction with vangl2, we designed two RNA-Seq experiments that largely overlapped in 127 design (Fig 2A-B). Embryos were exposed to a subteratogenic dose of 1\% ethanol in 128 embryo media (171 mM), which equilibrates to approximately $50 \mathrm{mM}$ tissue concentration 129 [17]. For the first experiment, embryos were treated at the onset of gastrulation (6 hpf) and 130 collected at mid-gastrulation ( $8 \mathrm{hpf}$ ) and the end of gastrulation (10 hpf) (Fig 2A). A second 131 experiment was performed to increase power (Fig 2B). Embryos were similarly exposed, 132 but a 14 hpf time window was included, when the eye fields have completely separated.

133 Each sample consisted of an individual zebrafish embryo with five replicates per timepoint

134 and treatment. The $6 \mathrm{hpf}$ control samples were omitted since they lacked ethanol-treated 135 samples for comparison. The data from both experiments were combined for subsequent 136 analyses, controlling for batch effects.

137 To assess the effect of time, batch (i.e. experiment 1 and 2), and ethanol, on the 138 early zebrafish transcriptome, we performed principal component analysis (PCA).

139 Individuals from each timepoint clustered tightly along $P C 1$, which accounted for $38 \%$ of

140 the transcriptional variation observed across the datasets. This strong effect of time on the 141 zebrafish transcriptome is in agreement with previous studies [18]. Clustering of samples 142 by time, regardless of ethanol treatment suggested that control and ethanol-treated samples 143 were accurately staged, indicating that ethanol did not delay developmentally regulated 144 transcriptome patterns. There was greater discrimination of $14 \mathrm{hpf}$ samples relative to 
145 earlier timepoints $(6,8$, and $10 \mathrm{hpf})$, which is consistent with greater distinction of this

146 timepoint in terms of developmental time and morphology (Fig 2C). PC2 largely captured

147 batch effects between experiment 1 and 2 (Fig 2D).

148 The majority of variation between samples did not appear to be due to treatment,

149 with control and ethanol-treated samples randomly interspersed along PC1 and PC2 (Fig

$1502 \mathrm{E})$. Separation by treatment was observed along $P C 8$ and $P C 9$, which accounts for $3 \%$ of

151 the variation in the data (Fig 2F). Hierarchical clustering of samples based on correlation,

152 further corroborated this finding. The 6 and 14 hpf samples showed the greatest

153 dissimilarity, whereas the 8 and $10 \mathrm{hpf}$ samples showed the greatest similarity, irrespective

154 of treatment (S1 Fig). In summary, the transcriptional effect of exposure to a subteratogenic

155 dose of ethanol on the early zebrafish transcriptome was subtle, while time provided the 156 strongest transcriptional fingerprint.

\section{Ethanol has effects on transcription that are largely distinct between different} 158 developmental timepoints

Although developmental age was a stronger source of transcriptional variation, we

160 still detected substantial variation in gene expression following subteratogenic ethanol

161 exposure. There were 1,414 differentially expressed genes (DEGs), with a false-discovery

162 rate (FDR) less than 0.1 (Fig 3A; Benjamini-Hochberg procedure). There were more

163 upregulated than downregulated DEGs among ethanol-treated individuals across

164 timepoints (S2 Fig) and these DEGs were distinct between developmental timepoints (Fig

$1653 \mathrm{~A}, \mathrm{~B})$. In summary, while some genes are generally affected by ethanol across several 166 critical periods, ethanol largely elicits unique responses at distinct developmental stages 167 spanning early embryogenesis. 


\section{Ethanol does not affect the Wnt/PCP pathway at the transcriptional level}

While vangl2 mutants do not typically exhibit cyclopia, cyclopia is observed in

170 double mutants between vangl2 and other Wnt/PCP pathway members [15, 19]. One

171 potential mechanism for the interaction between ethanol and vangl2 would be the

172 transcriptional misregulation of other Wnt/PCP pathway members. However, transcription

173 of Wnt/PCP pathway members was largely unaffected by ethanol exposure. No Wnt/PCP

174 pathway members were among the 14 shared DEGs across all timepoints (Fig 3C). KEGG

175 pathway enrichment analysis further confirmed that ethanol had little effect on the

176 Wnt/PCP pathway at the level of transcription (Fig 3D). Only two Wnt/PCP pathway

177 members were moderately affected: ethanol exposure moderately decreased expression of

178 the cofactor, glypican $4(g p c 4),\left(\log _{2}\right.$ fold $=-0.237$; -value $\left.=0.036\right)$ across all timepoints

$179[15,20]$ and upregulated expression of $r a c 3 a\left(\log _{2}\right.$ fold $=0.737$; p-value $\left.=8.89 \mathrm{E}-06\right)$, a

180 member of the Rho family of small GTPases [21]. We plotted the normalized read counts

181 across each time point from the RNA-seq experiment to further investigate the dynamics

182 of the potential alteration in $g p c 4$ levels. The expression of $g p c 4$ was modestly reduced in

183 ethanol-treated embryos across the RNA-seq dataset (Fig 3E). Ethanol exposure

184 consistently downregulated gpc4 at 8 and $10 \mathrm{hpf}$, but the magnitude of the downregulation

185 was relatively modest. To statistically compare ethanol-treated and control embryos,

186 expression of $g p c 4$ at $10 \mathrm{hpf}$ was investigated using qRT-PCR. This result demonstrated

187 that gpc4 was not significantly affected by ethanol exposure (Fig 3F). Thus, direct

188 transcriptional alteration to the Wnt/PCP pathway is unlikely to explain the ethanol-

189 induced phenotypes in vangl2 mutants and heterozygotes. 


\section{Modules of co-regulated genes related to ethanol exposure}

To determine if ethanol disrupted networks of genes that could explain the vangl2-

192 ethanol interaction, we next performed Weighted Gene Co-expression Network Analysis

193 (WGCNA) [22]. This unsupervised network analysis identifies groups of genes, termed

194 modules, based on correlated expression patterns across the samples. Modules are

195 summarized by the first principal component for the expression estimates of the included

196 genes, termed the module eigengene, which can be correlated with sample traits to identify

197 biological significance. The cluster dendrogram generated in this analysis illustrates the

198 presence of highly distinct and clustered modules (S3A Fig). Merging of similar modules

199 produced eleven total modules (S3B Fig). Consistent with our PCA analysis, the module

200 eigengenes were primarily correlated with time. We used these modules to further validate

201 our ethanol exposure regimen.

202 Previous work has shown that ethanol delays development in a dose-dependent 203 manner at concentrations equal to or greater than $1.5 \%$ ethanol [23]. For the experiments

204 herein, ethanol-treated samples were morphologically stage-matched to control samples to 205 exclude differences due to developmental age or delay. To confirm that the ethanol samples 206 were indeed age-matched to the control samples at the level of transcription, we compared 207 expression patterns of developmentally regulated genes between the ethanol and control 208 samples from each timepoint. For developmentally regulated genes, we used the gene with 209 the highest module membership (i.e. the hub gene) from each of the WGCNA modules that 210 was associated with time $(\mathrm{p}<0.05)(\mathrm{S} 4 \mathrm{Fig})$. Consistent with the results from the PCA (Fig $2112 \mathrm{C}$ ), the slope of the expression levels for each gene were similar in control and ethanol212 treated samples across age, most clearly demonstrated in the magenta4, thistle1, and 213 blueviolet modules (S4B,C,G Fig). Interestingly, we find a time-specific difference in the 214 expression of hub genes in the honeydew1, greenyellow, and saddlebrown modules at 10 
215 hpf (S4F,H,K Fig). Together, these data indicate samples were accurately age-matched and 216 the observed changes were biologically relevant.

217 Two modules (mediumpurple4 and darkolivegreen4) correlated with ethanol

218 treatment (S3B-D Fig). The purple and green modules positively and negatively correlated

219 with ethanol treatment, respectively (S3C Fig). A significant differentially expressed gene

220 from the green (S3G Fig) and purple module (S3H Fig) were selected for independent

221 validation on independent biological replicate samples derived from the same wild-type

222 zebrafish line using quantitative real-time RT-PCR (qRT-PCR). These results indicate that

223 our RNA-seq faithfully represents transcript levels. The green module is only weakly

224 downregulated and is enriched for genes encoding zinc finger $(\mathrm{ZnF})$ proteins, which

225 included znf1015 (S3E Fig). This module is almost entirely composed of genes on

226 chromosome 4, that have been shown to be co-regulated during these stages of

227 development $[24,25]$. The purple module revealed GO enrichment of transmembrane

228 transporters, which included slc16a9a (S3F Fig). As an upregulated module enriched in

229 transporters, these genes are likely to represent the physiological response to ethanol. Thus,

230 while we did identify two modules associated with ethanol ( $\mathrm{S} 1 \mathrm{Table}$ ), the module

231 membership did not provide clues to the interaction between ethanol and vangl2.

\section{Cyclopamine is predicted to mimic the effects of ethanol}

233 One challenge in RNA-seq analyses is inferring the mechanism underlying a

234 diseased or environmentally-perturbed state from a large set of differentially expressed

235 genes. Individual functional analyses of significant gene-ethanol interactions are time

236 consuming and thus inefficient. To circumvent this problem, we adopted a bioinformatics

237 approach, utilizing the Library of Integrated Network-Based Cellular Signatures (LINCS

238 L1000) toolkit [26]. This transcriptomic dataset includes gene expression data from nine 
239 human cancer cell lines exposed to thousands of small molecule drugs [26]. We queried 240 the top 100 and 150 up- and down-regulated genes induced by ethanol exposure against 241 the LINCS L1000 dataset using the clue.io platform (https://clue.io). The query generated

242 a list of small molecules predicted to have a positive or negative correlation to the input 243 signature (i.e. small molecules predicted to either mimic or antagonize the transcriptional 244 effects of ethanol). We were particularly interested in those chemicals with positive 245 correlation to ethanol as they would give insight into the mechanism of ethanol 246 teratogenicity and highlight potential co-factors that exacerbate ethanol teratogenicity.

247 Interestingly, cyclopamine, a hallmark Shh pathway inhibitor that inhibits the core 248 Shh pathway protein Smoothened (Smo), was predicted to positively correlate with the 249 ethanol signature. The Shh pathway is critical for midfacial development and mice 250 deficient in Sonic Hedgehog (Shh) exhibit severe brain and face malformations, including 251 holoprosencephaly and a single medial eye (cyclopia) [27]. In zebrafish, reduction of $s h h$

252 or null mutations in smo similarly results in severe loss of craniofacial midline structures 253 (i.e. the anterior neurocranium) [28]. Animal models have demonstrated ethanol is an 254 environmental risk factor for holoprosencephaly, resulting in a characteristic set of 255 midfacial defects, a hypomorphic forebrain, and in severe cases, cyclopia [16, 29-32]. 256 Thus, attenuation of the Shh pathway could mechanistically explain the vangl2-ethanol 257 interaction.

\section{Ethanol indirectly attenuates Shh signaling}

259 To investigate the interaction of cyclopamine and ethanol, we first exposed wild260 type zebrafish embryos to cyclopamine $(50 \mu \mathrm{M})$ at shield stage $(6 \mathrm{hpf})$ for 24 hours, 261 mimicking the ethanol exposure window for the vangl2 mutants. Embryos were fixed at 4 $262 \mathrm{dpf}$ and the cartilage and bone were stained with Alcian blue and Alizarin red, respectively. 
263 We observed a range of midfacial defects with the most severe phenotype being a complete

264 loss of the anterior neurocranium and reduced spacing between the eyes (Fig 4A). Since

265 cyclopamine was predicted to mimic the effects of ethanol, we next combined this low

266 dose of cyclopamine with the subteratogenic dose of ethanol. Strikingly, all embryos

267 presented with synophthalmia or cyclopia and significant reductions and defects of the

268 cartilages of the neuro- and viscerocranium. To quantify this combinatorial effect, we

269 measured the distance between the lenses and observed a significant reduction in co-

270 exposed embryos relative to those exposed to either ethanol or cyclopamine alone

$271(\mathrm{p}<0.0001)$, suggesting a strong synergistic interaction (Fig 4B).

272 In chick, ethanol exposure during somitogenesis has been proposed to suppress Shh

273 signaling and induce apoptosis in cranial neural crest cells that make up the craniofacial

274 skeleton [33]. However, work in zebrafish shows only a modest increase in cell death

275 within the eye field at a much higher dose of $2 \%$ ethanol [34]. To ensure that cells in the

276 eye field are not simply undergoing apoptosis, we performed a TUNEL cell death assay in

277 ethanol-treated vangl2 mutants at $11 \mathrm{hpf}$, prior to optic vesicle evagination. As expected,

278 we failed to detect an increase in apoptotic cells within the eye field in vangl2 mutants or

279 their siblings (S5 Fig). Thus, Shh-mediated pro-survival signals do not appear to be 280 significantly disrupted by ethanol.

281 To test for a direct effect of ethanol on Shh signaling, we quantified the relative 282 expression of ptch2, a canonical read-out of Shh pathway activity, at bud stage (10 hpf).

283 Subteratogenic doses of ethanol had no effect on the expression levels of ptch2, consistent

284 with RNA-seq results (Fig 4D). While cyclopamine significantly reduced Shh signaling (p $285=0.0006)$, ethanol did not further reduce $p t c h 2$ levels significantly $(\mathrm{p}=0.1115)$. KEGG 286 analysis from the RNA-seq confirmed that ethanol does not affect the Shh pathway as a 
287 whole, nor does it reduce the levels of Shh target genes (i.e. gli, ptch) (Fig 4E). Thus, at 288 normally subteratogenic doses, ethanol does not appear to directly attenuate Shh signaling.

\section{Ethanol disrupts convergent extension}

The ethanol-induced vangl2 mutant phenotype closely mirrors those in compound

291 mutants between vangl2 and other Wnt/PCP pathway members $[15,19]$. These double

292 mutants display a further reduction in convergent extension, as evidenced by a shorter and

293 broader body axis [15]. Based on these data, we hypothesized that ethanol disrupts

294 convergent extension, which would mislocalize the Shh signal and result in eye defects.

295 We performed in situ hybridization on untreated and ethanol-treated vangl2

296 embryos to examine convergent extension and to test the hypothesis that the Shh expression

297 domain is mislocalized in ethanol-exposed vangl2 mutants. We analyzed expression of $s h h$

298 in the axial mesoderm and paired box $2 a$ (pax $2 a)$ in the midbrain-hindbrain boundary at

299 bud stage (10 hpf) [35]. Ethanol was initiated at high stage (3.3 hpf), when vangl2

300 heterozygotes and homozygotes are equally sensitive to the effects of ethanol. We observed

301 a gene and ethanol-dose dependent reduction in the length of the shh expression domain

302 (extension) and an increase in the width of the pax $2 a$ expression domain (convergence)

303 (Fig 5A).

304 To quantify the effect of ethanol on convergent extension, we plotted the 305 normalized expression values of shh/pax2 (Fig 5B). Post-hoc analyses (Tukey's) revealed 306 significant differences between untreated embryos across vangl2 genotypes, indicating that

307 vangl2 gene dosage affects convergent extension. Similarly, we found significant 308 differences between ethanol-treated mutants and their heterozygous and wild-type siblings $309(\mathrm{p}=0.017)$. This observation confirms that loss of vangl2 results in reduced convergent 310 extension movements, as evidenced by their short body axis. This phenotype was 
311 exacerbated with ethanol treatment, resulting in reduced convergent extension for vangl2

312 heterozygotes and homozygotes, compared to their untreated counterparts. While there was

313 a trend, we did not observe a difference in convergent extension between ethanol-treated

314 vangl2 heterozygotes and their wild-type siblings in their shh/pax2 ratio. However,

315 synophthalmia occurred in ethanol-treated heterozygotes but not wild-type embryos,

316 demonstrating that the nonsignificant reduction in convergent extension in the

317 heterozygotes can have a phenotypic consequence.

\section{Ethanol alters six 3 and $r x 3$ expression in the eye field}

319 Transcription factors involved in the specification of the eye field have also been

320 implicated in the mechanism of eye field separation. The expression of three of these

321 transcription factors, six 3, rx3, and $r x 1$, are altered by ethanol exposure [34]. We examined

322 the effect of ethanol on six $3 a$ and $r \times 3$ in ethanol-treated vangl2 mutants at the initiation of

323 optic vesicle evagination. In situ hybridization of six $3 a$ in $11 \mathrm{hpf}$ embryos shows a heart-

324 shaped expression pattern in the prospective forebrain. The caudal indentation marks the

325 splitting of the eye field into bilateral domains (Fig 5C). This expression pattern becomes

326 more diffuse with loss of vangl2. In untreated homozygous mutants, we observed a

327 shortening along the anterior-posterior (AP) axis and a broadening along the mediolateral

328 axis at $11 \mathrm{hpf}$, consistent with the convergent extension defect. This expression pattern was

329 further exacerbated in ethanol-treated mutants with complete loss of the caudal indentation.

330 We observed a similar effect of genotype and ethanol on $r x 3$ expression at mid-evagination

331 (12 hpf) (S6 Fig). At this stage, $r x 3$ is localized to the prospective forebrain and retina [36].

332 Ethanol-treated vangl2 homozygous mutants exhibit a compressed expression domain,

333 clearly displaying a reduction in convergent extension. Our expression analyses suggest 
334 the eye field is specified but mis-localized and fails to separate into bilateral domains, likely

335 due to defects in mesodermal migration.

336 Mutation in gpc4 enhances cyclopia in a dose-dependent manner

337 Our data support a model in which ethanol interacts with vangl2 via a combinatorial

338 disruption of convergent extension. If ethanol disrupts convergent extension, which leads

339 to interactions with vangl2, then further genetic disruption to convergent extension should

340 exacerbate the ethanol-vangl2 phenotype. Embryos deficient in gpc4 similarly have a

341 defect in convergent extension as evidenced by their shortened, broadened body axis [15].

342 Previous work in zebrafish has shown a functional interaction between vangl2 and gpc4,

343 where vangl2;gpc4 double mutants were invariably cyclopic [15]. We conducted

344 additional functional analyses to further examine the relationship between these two genes

345 in the context of ethanol exposure. Consistent with Marlow et al., double mutants were

346 fully penetrant for cyclopia with or without ethanol. While $1 \%$ ethanol exposure altered

347 the facial morphology of gpc4 mutants (Fig 6A), it did not cause cyclopia. However,

348 ethanol concentrations greater than 1\%, which did not cause cyclopia in wild-type siblings,

349 resulted in cyclopia in gpc4 mutants (S7 Fig) [15]. Additionally, embryos carrying 3

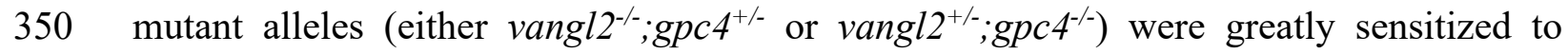

351 cyclopia when exposed to $1 \%$ ethanol (Fig 6B). Collectively these data indicate that in

352 sensitized genotypes, ethanol disrupts convergent extension, thus mispositioning a source

353 of Shh that is essential for separation of the eye field into bilateral primordia.

354 Blebbistatin phenocopies the ethanol-induced defect in vangl2 mutants.

355 If ethanol interacts with the Wnt/PCP pathway via convergent extension, then 356 replacing ethanol with an inhibitor that disrupts cell movements, should similarly result in 
357 synophthalmia and/or cyclopia. We extended our analyses to test the effect of blebbistatin,

358 a myosin II inhibitor that reduces axis elongation, in vangl2 mutants [37]. Blebbistatin

359 treatment during gastrulation $(6-10 \mathrm{hpf})$ induced synophthalmia in vangl2 heterozygotes

360 and homozygotes (Fig 7A). We then quantified this interaction by measuring the inner lens-

361 to-lens width and found a significant reduction in blebbistatin-treated vangl2 homozygotes

362 (Fig 7B). Furthermore, 26.92\% and 100\% of blebbistatin-treated heterozygotes and

363 homozygotes, respectively, were cyclopic (Fig 7C). These data suggest ethanol further

364 inhibits convergent extension in the sensitized background, vangl2, which not only disrupts

365 their ability to elongate their body axis, but also inhibits the splitting of the eye field.

\section{Ethanol affects vangl2 filopodia organization on migrating cells}

367 Based on our data, cell dynamics underlying convergent extension are likely 368 involved in the vangl2-ethanol interaction. During gastrulation, mesodermal cells adopt a 369 bipolar shape and orient their actin-based cytoskeletal projections on their leading and 370 trailing edge, with respect to the notochord, allowing for proper convergent extension [38].

371 In vangl2 mutant embryos, these gastrula cells fail to elongate and align with the primary 372 axis [39]. Furthermore, vangl2 mutant ectodermal cells possess a greater number of 373 filopodial protrusions than wild-type siblings and display projections in an unpolarized 374 manner [40].

375 To determine whether ethanol may be exacerbating this phenotype, we analyzed 376 filopodia protrusion length and orientation around the circumference of migrating live 377 mesodermal cells. We injected memGFP mRNA into a single blastomere of vangl2 378 embryos at the 8-32 cell stage, to obtain mosaic expression. We then treated a subset of the 379 injected embryos with 1\% ethanol from 6-10 hpf. Homozygous mutants were identified 380 based on their reduced body axis elongation at $10 \mathrm{hpf}$. Cells were imaged at $40-60^{\circ}$ from 
381 the primary axis. Spike-like filopodia were highly unpolarized in ethanol-treated vangl2 382 mutants compared to their wild-type and heterozygous siblings (Figure 8A). We quantified 383 the polarity and number of filopodia for each cell, with respect to the head $\left(90^{\circ}\right)$, the 384 primary axis/notochord $\left(180^{\circ}\right)$, and the tail $\left(270^{\circ}\right)$ (Figure $\left.8 \mathrm{~B}\right)$. Rose diagrams suggest 385 ethanol-treated vangl2 mutants have more un-polarized projections on the 386 anterior/posterior edge (red) as opposed to the leading (green) or trailing (blue) edge. We

387 next reduced the number of bins in the rose diagrams to $90^{\circ}$ and noticed a clear over388 representation of filopodia in vangl2 mutants on their anterior/posterior edge (Figure 8C).

389 Tukey's post-hoc analyses confirmed ethanol-treated vangl2 mutants had significantly

390 more filopodia on their anterior/posterior edge compared to other axes and their wild-type 391 or heterozygous siblings (Figure 8D). These data confirm that ethanol exposure is 392 associated with an increase in the number of filopodial projections oriented in the wrong 393 direction, or perpendicular to the path of dorsal migration, in ethanol-treated vangl2 394 mutants.

\section{DISCUSSION}

396 Molecular staging for the early zebrafish embryo

397 Despite their importance, the nature and mechanisms of gene-environment 398 interactions are largely unknown. Here, we performed single embryo bioinformatic 399 analyses to shed light upon the mechanism behind gene-environment interactions. A 400 growing number of researchers are using the zebrafish for toxicological and/or gene401 environment studies. One potential confounding variable in these types of studies is 402 developmental delay. Our WGCNA analyses are rich in gene modules that similarly vary 403 over developmental time, providing a way to molecularly stage embryos. Thus, our results 404 will provide important resources to ensure the rigor and reproducibility of these analyses. 
The effects of ethanol on the transcriptome is subtle.

Our hierarchical clustering analysis demonstrates that the effect of ethanol on the 407 transcriptome is minor, within any given time point ethanol-exposed and unexposed 408 embryos intermingled. PCA results support this, demonstrating that only $3 \%$ of the

409 variance in the transcriptomic data is attributable to ethanol exposure. In sum, we found 4101,414 differentially regulated genes across all time points with the majority of these genes 411 being upregulated. Most differentially expressed genes were specific to a single time point.

412 At any given time point there were between 92 and 328 differentially expressed genes.

413 Thus, exposure to a normally subteratogenic dose of ethanol results in subtle and dynamic 414 alterations to the transcriptome.

415 Our findings identified dramatically fewer differentially expressed genes than those 416 observed in transcriptomic analyses of ethanol-exposed chicken and mouse embryos. In 417 chicken 3,422 differentially regulated genes were observed after 6 hours of ethanol 418 treatment and the preponderance (1,924 genes) were downregulated [41]. In a mouse 419 microarray, 2,906 differentially expressed genes were identified 3 hours after ethanol

420 injection [42]. Species-specific developmental trajectories or the tissue analyzed, cranial 421 neural fold (chicken), headfold (mouse), versus whole embryo (our study) may account for 422 some of these differences. However, it is important to note that the chicken and mouse 423 strains analyzed are highly sensitive to the dose of ethanol used while our zebrafish $\mathrm{AB}$ 424 strain is not (without the addition of a sensitizing mutation). Thus, the chicken and mouse 425 data likely represent transcriptomic changes representative of pathogenesis. Our zebrafish 426 data reflect a direct effect of ethanol, which may or may not be pathogenic depending upon 427 the genetic and environmental milieu.

428 WGCNA analysis identified two modules (mediumpurple4 and darkolivegreen4) 429 of genes that are coordinately altered by ethanol exposure. The vast majority of genes that 
430 negatively correlate with ethanol exposure (membership in darkolivegreen4) encode zinc 431 finger proteins $(\mathrm{ZnF})$ located on chromosome 4. Ethanol has similarly been shown to 432 downregulate Zinc finger protein, subfamily 1A, 4 in mouse fetuses exposed to ethanol

433 during early development [43]. However, the functional significance of these changes in $434 \mathrm{ZnF}$ proteins remains unknown and they often have diverse binding affinities and 435 functions. The long arm of zebrafish chromosome $4(\mathrm{Chr} 4 q)$ is typically heterochromatic 436 (condensed) and lacking in protein-coding genes [44]. However, it is not late replicating 437 until the end of gastrulation or bud stage (10 hpf) [25]. Previous work in zebrafish has 438 found $\mathrm{ZnF}$ proteins on chromosome 4 to undergo robust expression from the initiation of 439 zygotic transcription until mid-gastrula stage [24]. Since many chromosome 4 genes are 440 downregulated across ethanol-treated individuals during gastrulation, ethanol may 441 interfere with replication timing, blocking the early-to-late replication switch, or chromatin 442 remodeling. Based on these findings, the differentially expressed genes may follow 443 disrupted expression of $\mathrm{ZnF}$ proteins due to chromatin remodeling.

\section{Ethanol interacts with vangl2 through the indirect effects on the Shh pathway}

445 Because Vang12 is a core member of the Wnt/PCP pathway, a simple explanation

446 for ethanol-sensitivity in vangl2 mutants would be that ethanol transcriptionally 447 dysregulates the Wnt/PCP pathway. However, our RNA-seq results indicated that this is 448 not the case. The expression of gpc4 was the only member of the Wnt/PCP pathway that 449 was downregulated in our RNA-seq dataset. Cyclopia is observed in vangl2;gpc4 double 450 mutants [15] and we show that heterozygosity for gpc4 further sensitized vangl2 mutants 451 to ethanol. However, the downregulation of gpc4 was quite modest and unlikely to have 452 any substantial impact on the phenotype of ethanol-exposed vangl2 mutant.

453 We used an unbiased approach, the LINCS L1000 dataset to identify pathways that 454 might be disrupted by ethanol and generate cyclopia in vangl2 mutants. One of the top 
455 matches to the effects of ethanol was cyclopamine, a Hh pathway inhibitor. Shh signaling 456 is critically important for development of the midface across species [27, 45-47]. In 457 zebrafish shha and smo mutants have cyclopia because Shh signaling to the diencephalon

458 is critical for separation of the eye fields [48, 49]. Furthermore, cyclopia observed in 459 Wnt/PCP pathway mutants is due to the mispositioning of this source of Shh $[16,19]$. Thus, 460 disruption of the Shh pathway could mechanistically explain the ethanol sensitivity of 461 vangl2 mutants.

462 The Shh pathway has been implicated in ethanol teratogenesis. However, there has 463 been much controversy regarding whether this interaction is direct or not [8]. As predicted 464 by LINCS, we find a synergistic interaction between ethanol and the Hh pathway inhibitor 465 cyclopamine. However, both our RNA-seq and qPCR data demonstrate that, at this timing 466 and dose, ethanol does not alter the expression of direct readouts of Shh activity. Such an 467 alteration would be expected if ethanol interacted directly with core components of the 468 pathway. As supported by our in situ analysis, we conclude that ethanol indirectly effects 469 the Shh pathway by mislocalizing the source of the ligand.

\section{Ethanol disrupts convergent extension by disrupting polarized cellular protrusions.}

471 The phenotypes observed in ethanol-exposed vangl2 mutants suggest that ethanol 472 further disrupts convergent extension. Our findings are consistent with previous 473 observations that high doses of ethanol can generate phenotypes consistent with a 474 disruption of convergent extension in zebrafish and Xenopus [16, 50, 51]. More recently, 475 zebrafish microarray analyses demonstrated that disrupted gastrulation movements due to 476 ethanol exposure were caused by a reduction in pchd18a expression [52]. The expression 477 of pchd18a is not altered in our dataset. However, Sarmah and colleagues used the ethanol478 sensitive TL strain and exposed embryos to ethanol from 2 to $8 \mathrm{hpf}$. This later difference 479 is particularly intriguing giving our finding that the time window of greatest sensitivity to 
480 ethanol was different for vangl2 heterozygotes and mutants, with heterozygotes being most 481 sensitive at $3.3 \mathrm{hpf}$ (the earliest time we tested). This raises the intriguing possibility that 482 the earlier time window of teratogenesis is due to a different mechanism.

483 We propose that ethanol leads to midfacial defects and cyclopia, not by 484 dysregulating Wnt/PCP at the level of transcription, but indirectly through inhibition of 485 convergent extension and mislocalization of important developmental regulators like shha. 486 At the start of convergent extension, lateral gastrula cells migrate and converge to the 487 dorsal region of the developing embryo, where they intercalate between neighboring cells 488 to drive extension of the body axis $[20,53,54]$. As these cells begin their dorsal migration, 489 they elongate along their mediolateral axis and polarize their actin-based cytoskeletal 490 processes medially and laterally to drive intercalation [54, 55]. Convergent extension 491 movements during gastrulation also alter the shape of the eye field, whereby diencephalic 492 precursor cells articulate both medially and caudally [56]. We know from previous work 493 that vangl2 mutant lateral gastrula cells fail to elongate their mediolateral axis and have a 494 slight anterior bias in their dorsal trajectory $[39,57]$. In addition to their shape changes, 495 vangl2 mutant ectodermal cells have more filopodia than wildtype cells and defective 496 polarization (i.e. less filopodia localized on the trailing edge), resulting in an indirect dorsal 497 trajectory [40]. Using live confocal imaging coupled with memGFP expression, we 498 examined the effect of ethanol on filopodia number and polarity in vangl2 embryos. 499 Interestingly, we did not find more filopodia in vangl2 lateral mesodermal cells relative to 500 wildtype cells. Despite this, we find that ethanol-treated vangl2 mutant embryos have more 501 filopodia on their anterior/posterior edge relative to their mediolateral edge. In support of 502 our hypothesis, we demonstrate that ethanol disrupts protrusion polarity, convergent 503 extension, and shha localization, with greater disruption observed in vangl2 mutants. 


\section{MATERIALS AND Methods}

\section{Zebrafish (Danio rerio) Care and Use}

506 Zebrafish were cared for using standard IACUC-approved protocols at the

507 University of Texas at Austin. The wild-type AB strain was used for RNA-seq analysis.

508 The vangl2 ${ }^{m 209}$ allele, originally described as $\operatorname{tri}^{m 209}$ [39], was obtained from the Zebrafish

509 International Resource Center (ZIRC) as reported [13]. The gpc $4^{\text {fr } 6}$ line was provided by

510 Dr. Lila Solnica-Krezel. Adult fish were maintained on a 14h/10h light-dark cycle at

$51128.5^{\circ} \mathrm{C}$. Embryos were collected and staged according to morphology and somite number $512 \quad[58]$.

\section{Chemical treatments}

514 Embryos were treated with 1\% ethanol diluted in embryo media. This 515 subteratogenic dose mimics an acute (binge-like) alcohol exposure roughly equivalent to a 516 blood alcohol concentration of $0.19 \%$ in humans. AB embryos were treated with $50 \mu \mathrm{M}$

517 cyclopamine (Toronto Research Chemicals C988400), diluted in embryo media. The 518 concentration of ethanol (vehicle) in the embryo media was controlled for between 519 treatment groups and equals a final concentration of $0.5 \%$ in the EM. Blebbistatin (Sigma520 Aldrich B0560) was dissolved in DMSO and diluted to a concentration of $37.5 \mu \mathrm{M}$ in 3 $521 \mathrm{~mL}$ of embryo media. Following chemical exposure, embryos were washed 3x in embryo 522 media and then grown to $4 \mathrm{dpf}$ before fixation. memGFP cloned in the pCS2 vector was linearized by NotI restriction 525 endonuclease. Synthetic mRNA was generated using the mMESSAGE mMACHINE ${ }^{\mathrm{TM}}$ 
526 SP6 Transcription Kit (Invitrogen, AM1340). Embryos were microinjected at 100pg

527 between the $8-16$ cell stage [40].

\section{Sample collection and RNA extraction}

529 Single embryos were manually dechorionated and collected in a $1.75 \mathrm{~mL}$ 530 microcentrifuge tube with $500 \mathrm{~mL}$ of TRIzol reagent (Life Technologies, 15596-026). 531 Embryos were homogenized with a motorized pestle (VWR, 47747-370) and stored at $53280^{\circ} \mathrm{C}$ until RNA extraction. Total RNA was processed according to the TRIzol RNA 533 isolation protocol. Samples were re-suspended with $50 \mu \mathrm{L}$ of nuclease-free water and 534 subsequently purified using the RNA Clean \& Concentrator kit (Zymo, R1018). The 535 concentration of each sample was determined using a Nanodrop spectrophotometer. The 536 quality of total RNA was analyzed with the Agilent BioAnalyzer to ensure that the RNA 537 Integrity Number (RIN) was $\geq 8$. Samples were submitted to the Genomic Sequencing and 538 Analysis Facility (GSAF) at the University of Texas at Austin. The GSAF performed 539 standard RNA-Seq library preparations with poly-A mRNA capture.

\section{$540 \quad$ RNA-seq data processing}

541 Sequencing on the NextSeq 500 platform produced an average of 40.8 million \pm

5421.4 million (SE) raw paired end reads per sample. Adapter trimming was performed using 543 Cutadapt with a minimum length of 25 bp [59]. Following adapter trimming, we retained 544 an average of $40.0 \pm 1.3$ million (SE) reads per sample. Genome Reference Consortium 545 Zebrafish Build 10 (GRCz10) for D. rerio was downloaded from Ensembl [60]. Trimmed 546 reads were mapped to the reference using STAR [61]. Mean mapping efficiency was 78.2\% $547 \pm 0.8 \%$ (SE). Following mapping PCR duplicates were removed using Picard 548 (https://broadinstitute.github.io/picard/). Duplication rate was estimated at $85 \% \pm 0.8 \%$ 
549 (SE). Sorting and conversion between SAM and BAM files was performed using samtools

550 [62]. Reads mapping to annotated genes were counted with HTseq version 0.6.1p1 using

551 the intersection nonempty mode [63]. The final number of reads mapped to annotated genes

552 was on average $3.9 \pm 0.2$ million reads per sample. Detailed instructions and example

553 commands for implementing the data processing steps described above are available on

554 Github (https://github.com/grovesdixon/Drerio_early_ethanol_RNAseq).

\section{Differential expression analysis}

556 Normalization and statistical analysis of read counts was performed using DESeq2

557 [64]. Factors included in the differential expression models were ethanol treatment

558 (control, treated), developmental timepoint (8 hpf, $10 \mathrm{hpf}$, and $14 \mathrm{hpf}$ ), and sequencing

559 batch (experiment 1 or experiment 2). Because none of the 6 hpf samples were treated

560 with ethanol, these samples were not included. We tested for differential expression 561 associated with ethanol treatment using likelihood ratio tests-comparing the model

562 including all three factors to a reduced model that did not include ethanol treatment. To

563 further examine stage-specific ethanol effects we split the samples by developmental

564 timepoint and tested for ethanol effects within each group.

\section{Weighted Gene Correlation Network Analysis (WGCNA)}

566 Gene expression data were further analyzed with Weighted Gene Correlation 567 Network Analysis (WGCNA) [22]. For input into the analysis, we used variance stabilized 568 counts generated using the rlog function in DESeq2 [64]. Genes that were not sequenced 569 across sufficient samples (4320 in total) were removed using the goodSamplesGenes 570 function in the WGCNA package. Because there were no ethanol treated samples for the 571 six hour timepoint, the five samples from this timepoint were removed before further 
572 analysis. To ensure sufficient expression for correlation detection, genes were further

573 filtered based on a base mean expression cutoff of 5. We controlled for batch effects using

574 the ComBat function from the R package sva [65]. We selected a soft threshold of 15,

575 where the scale free topology model fit surpassed 0.8. WGCNA was run with a minimum

576 module size of 10 . Following network analysis, we tested for GO enrichment within

577 modules using Fisher's exact tests.

\section{GO enrichment analysis}

579 Enrichment of Gene Ontology (GO) terms for ethanol responsiveness was tested 580 using two-tailed Mann-Whitney U-tests [66] followed by Benjamini-Hochberg procedure 581 for false discovery correction [67]. The results were plotted as a dendrogram tracing 582 hierarchical relationships between significant GO terms. The direction of enrichment (for 583 upregulation or downregulation) was indicated by text color and significance of enrichment 584 by font type. An advantage of this approach is that it does not require an arbitrary cutoff to 585 provide counts of "significant" and "non-significant" genes as in typical enrichment tests.

\section{Quantitative Real-Time RT-PCR (qRT-PCR)}

587 To validate our RNA-seq data, we selected two genes to test using qRT-PCR. Total 588 RNA was reverse transcribed using SuperScript ${ }^{\mathrm{TM}}$ First-Strand Synthesis System for RT589 PCR (Invitrogen) with oligo-d(T) primers. qRT-PCR was performed with Power Sybr 590 Green PCR Master Mix (Thermo Fisher Scientific, 4367659) on the Applied Biosystems 591 ViiA 7 Real-Time PCR System. QuantStudio Real-Time PCR Software was used for 592 data analysis using the $2^{-\Delta \Delta \mathrm{Ct}}$ method. The endogenous control $l s m 12 b$ was selected based 593 on its stable expression profiles across treatment and stage groups in the RNA-seq datasets. 


\section{KEGG enrichment methods and results}

We tested for enrichment of significant upregulated and downregulated genes among KEGG pathways separately using Fisher's exact tests. For each KEGG pathway,

597 we tested a two-way contingency table with inclusion in the KEGG pathway as columns, 598 and significant (FDR < 0.05) upregulation or downregulation as rows. P-values were then 599 adjusted for multiple tests using Benjamini Hochberg Method [67].

600 Three KEGG pathways were enriched for significant differential expression. Two 601 for upregulated genes, and one for downregulated genes. The upregulated KEGG pathways 602 (dre00250 and dre00480) were (1) Alanine, aspartate and glutamate metabolism, and (2) 603 Glutathione metabolism. The downregulated KEGG pathway (dre03013) was RNA 604 transport. The pathview figures for these are in 605 kegg_pathways/significant_pathway_figures/ in the git repository.

\section{Cartilage and Bone Staining and Measurements}

607 Embryos were fixed at 4 days post fertilization (dpf) and stained with Alcian blue 608 for cartilage and Alizarin red for mineralized bone [68]. Whole-mounts of vangl2 ${ }^{\text {m209 }}$ 609 embryos were captured using a Zeiss Axio Imager A1 microscope. To assess the degree of 610 cyclopia, the distance between the medial edges of the lenses was measured using the 611 AxiovisionLE software.

\section{In Situ Hybridization}

613 Antisense digoxygenin-labeled riboprobes for shha, pax2, dlx3, six3a and $r \times 3$

614 (together as a gift from Dr. Steve Wilson) were used. Whole-mount in situ hybridization 615 was performed as described [69]. Images were captured using the Zeiss Axio Imager A1 616 and expression domains were measured using the AxiovisionLE software. An ANOVA 617 and post-hoc Tukey's test were used for statistical analyses. 


\section{TUNEL Staining}

619 Whole-mount TUNEL staining was based on previous characterizations [12]. 620 Samples were fixed overnight in $4 \%$ paraformaldehyde in PBS (PFA) at $4^{\circ} \mathrm{C}$. Samples

621 were dehydrated in methanol and subsequently rehydrated in phosphate-buffered saline 622 containing $0.5 \%$ Triton X-100 (PBTx). Samples were permeabilized with $25 \mathrm{ug} / \mathrm{mL}$ 623 proteinase $\mathrm{K}(1 \mathrm{mg} / \mathrm{ml})$ in PBT for $30 \mathrm{~min}$. After two, 5 min washes with PBTx, samples

624 were fixed with 4\% PFA for $20 \mathrm{~min}$ at room temperature. Residual PFA was removed with 625 four, 5 min washes of PBTx. Samples were incubated with $50 \mu 1$ of 1:10 Enzyme:TUNEL 626 reagent (TdT and fluorescein-dUTP) (Roche, Cat No. 11684795910) at $37^{\circ} \mathrm{C}$ for $3 \mathrm{~h}$ in the 627 dark. The reaction was stopped with two, 5 min washes of PBTx. Confocal images were 628 captured with a Zeiss LSM 710.

\section{Confocal Imaging}

630 Control and ethanol-treated (6-10 hpf) embryos were dechorionated and staged at 63110 hpf. Homozygous mutants were distinguished between wild-type and heterozygotes by 632 their body axis elongation at bud stage. Live embryos were mounted in methyl cellulose. 633 Images were acquired using a Zeiss LSM710 confocal microscope using a 10x and 60x 634 objective.

\section{Quantification of filopodia}

636 Number of filopodia was acquired through projections of Z-stacks using the 60x 637 objective on the Zen software. Angle of projections were calculated using Fiji.

638 Rose diagrams of the mean number of filopodia found were plotted using 639 geom_bar() and the coord_polar() functions from ggplot2. Mean numbers of filopodia for 640 different embryonic regions were compared between groups using ANOVA and Tukey’s 
641 'Honest Significant Difference' method. Significantly distinct means were assigned based

642 on the Tukey's 'Honest Significant Difference' results using the R package multcomp [70].

\section{ACKNOWLEDGEMENTS}

644 We thank Dr. Steve Wilson for his kind contributions of the six3a and $r x 3$ riboprobes and

645 Dr. John Wallingford for his contributions of the memGFP plasmid. We also thank Dr. Lila

646 Solnica-Krezel for providing the $g p c 4^{f r 6}$ line. We are grateful to Anna Louise Percy, Angie

647 Martinez, and Cadianna Cotham, for maintenance and care of all zebrafish lines. Lastly,

648 we are grateful to our former undergraduate researchers, Jenna Beam, Elaine Avshman,

649 and Jennyly Nguyen, for their assistance in the lab.

650 


\section{Bibliography}

652 1. Czeizel AE. Birth defects are preventable. Int J Med Sci. 2005;2(3):91-2. Epub

653 2005/07/12. doi: 10.7150/ijms.2.91. PubMed PMID: 16007259; PubMed Central PMCID: 654 PMCPMC1168872.

$6552 . \quad$ Shepard TH. Detection of human teratogenic agents. The Journal of Pediatrics. $6561982 ; 101(5): 810-5$.

657 3. Harada M. Minamata Disease: Methylmercury Poisoning in Japan Caused by 658 Environmental Pollution. Critical Reviews in Toxicology. 1995;25(1):1-24.

659 4. Kim JH, Scialli AR. Thalidomide: the tragedy of birth defects and the effective 660 treatment of disease. Toxicol Sci. 2011;122(1):1-6. Epub 2011/04/22. doi: 661 10.1093/toxsci/kfr088. PubMed PMID: 21507989.

662 5. May PA, Gossage JP, Kalberg WO, Robinson LK, Buckley D, Manning M, et al. 663 Prevalence and Epidemiologic Characteristics of FASD from Various Research Methods 664 with an Emphasis on Recent Inschool Studies Developmental Disabilities 2009;15:176-92.

6656 Jones KL, Smith DW. Recognition of the Fetal Alcohol Syndrome in Early Infancy. 666 The Lancet. 1973:999-1001.

667 7. McCarthy N, Sidik A, Bertrand JY, Eberhart JK. An Fgf-Shh signaling hierarchy 668 regulates early specification of the zebrafish skull. Dev Biol. 2016;415(2):261-77. Epub 669 2016/04/10. doi: 10.1016/j.ydbio.2016.04.005. PubMed PMID: 27060628; PubMed 670 Central PMCID: PMCPMC4967541.

671 8. Eberhart JK, Parnell SE. The Genetics of Fetal Alcohol Spectrum Disorders. 672 Alcohol Clin Exp Res. 2016;40(6):1154-65. Epub 2016/04/29. doi: 10.1111/acer.13066. 673 PubMed PMID: 27122355; PubMed Central PMCID: PMCPMC5125635.

$6749 . \quad$ Streissguth AP, Dehaene P. Fetal alcohol syndrome in twins of alcoholic mothers: 675 concordance of diagnosis and IQ. Am J Med Genet. 1993;47(6):857-61. Epub 1993/11/01. 676 doi: 10.1002/ajmg.1320470612. PubMed PMID: 8279483.

677 10. Downing C, Balderrama-Durbin C, Broncucia H, Gilliam D, Johnson TE. Ethanol 678 teratogenesis in five inbred strains of mice. Alcohol Clin Exp Res. 2009;33(7):1238-45. 679 Epub 2009/04/25. doi: 10.1111/j.1530-0277.2009.00949.x. PubMed PMID: 19389189; 680 PubMed Central PMCID: PMCPMC2951264.

681 11. Loucks E, Carvan MJ, 3rd. Strain-dependent effects of developmental ethanol 682 exposure in zebrafish. Neurotoxicol Teratol. 2004;26(6):745-55. Epub 2004/09/29. doi: 683 10.1016/j.ntt.2004.06.017. PubMed PMID: 15451039. 
684 12. Lovely CB, Fernandes Y, Eberhart JK. Fishing for Fetal Alcohol Spectrum 685 Disorders: Zebrafish as a Model for Ethanol Teratogenesis. Zebrafish. 2016;13(5):391-8. 686 Epub 2016/05/18. doi: 10.1089/zeb.2016.1270. PubMed PMID: 27186793; PubMed 687 Central PMCID: PMCPMC5035362.

688 13. Swartz ME, Wells MB, Griffin M, McCarthy N, Lovely CB, McGurk P, et al. A 689 screen of zebrafish mutants identifies ethanol-sensitive genetic loci. Alcohol Clin Exp Res. 690 2014;38(3):694-703. Epub 2013/10/30. doi: 10.1111/acer.12286. PubMed PMID: 691 24164477; PubMed Central PMCID: PMCPMC3959233.

692 14. Solnica-Krezel L, Stemple DL, Mountcastle-Shah E, Rangini Z, Neuhauss SCF, 693 Malicki J, et al. Mutations affecting cell fates and cellular rearrangements during 694 gastrulation in zebrafish. Development. 1996;123:67-80.

695 15. Marlow F, Zwartkruis F, Malicki J, Neuhauss SCF, Abbas L, Weaver M, et al. 696 Functional Interactions of Genes Mediating Convergent Extension, knypek and trilobite, 697 during the Partitioning of the Eye Primordium in Zebrafish. Developmental Biology. 698 1998;203:382-99.

699 16. Blader P, Strahle U. Ethanol Impairs Migration of the Prechordal Plate in the 700 Zebrafish Embryo. Developmental Biology. 1998;201:185-201.

701 17. Lovely CB, Nobles RD, Eberhart JK. Developmental age strengthens barriers to 702 ethanol accumulation in zebrafish. Alcohol. 2014;48(6):595-602. Epub 2014/07/12. doi: 703 10.1016/j.alcohol.2014.06.003. PubMed PMID: 25012627; PubMed Central PMCID: 704 PMCPMC4163099.

705 18. Farrell JA, Wang Y, Riesenfeld SJ, Shekhar K, Regev A, Schier AF. Single-cell 706 reconstruction of developmental trajectories during zebrafish embryogenesis. Science. 707 2018;360(6392).

708 19. Heisenberg C-P, Tada M, Rauch G-Jr, Saude L, Concha ML, Geisler R, et al. 709 Silberblick/Wnt11 mediates convergent extension movements during zebrafish 710 gastrulation. Nature. 2000;405:76-81.

711 20. Topczewski J, Sepich DS, Myers DC, Walker C, Amores A, Lele Z, et al. The 712 Zebrafish Glypican Knypek Controls Cell Polarity during Gastrulation Movements of 713 Convergent Extension. Developmental Cell. 2001;1:251-64.

714 21. White JJ, Mazzeu JF, Coban-Akdemir Z, Bayram Y, Bahrambeigi V, Hoischen A, 715 et al. WNT Signaling Perturbations Underlie the Genetic Heterogeneity of Robinow 716 Syndrome. Am J Hum Genet. 2018;102(1):27-43. Epub 2017/12/26. doi: 717 10.1016/j.ajhg.2017.10.002. PubMed PMID: 29276006; PubMed Central PMCID: 718 PMCPMC5777383. 
22. Langfelder P, Horvath S. WGCNA: an R package for weighted correlation network analysis. BMC Bioinformatics. 2008;9:559. Epub 2008/12/31. doi: 10.1186/1471-2105-9559. PubMed PMID: 19114008; PubMed Central PMCID: PMCPMC2631488.

23. Loucks EJ, Ahlgren SC. Deciphering the role of Shh signaling in axial defects produced by ethanol exposure. Birth Defects Res A Clin Mol Teratol. 2009;85(6):556-67. Epub 2009/02/25. doi: 10.1002/bdra.20564. PubMed PMID: 19235835. resolution mRNA expression time course of embryonic development in zebrafish. Elife. 2017;6. Epub 2017/11/17. doi: 10.7554/eLife.30860. PubMed PMID: 29144233; PubMed Central PMCID: PMCPMC5690287.

729 25. Siefert JC, Georgescu C, Wren JD, Koren A, Sansam CL. DNA replication timing during development anticipates transcriptional programs and parallels enhancer activation. Genome Res. 2017;27(8):1406-16. Epub 2017/05/18. doi: 10.1101/gr.218602.116. PubMed PMID: 28512193; PubMed Central PMCID: PMCPMC5538556.

26. Subramanian A, Narayan R, Corsello SM, Peck DD, Natoli TE, Lu X, et al. A Next Generation Connectivity Map: L1000 Platform and the First 1,000,000 Profiles. Cell. 2017;171(6):1437-52 e17. Epub 2017/12/02. doi: 10.1016/j.cell.2017.10.049. PubMed PMID: 29195078; PubMed Central PMCID: PMCPMC5990023.

737 27. Chiang C, Litingtung Y, Lee E, Young KE, Corden JL, Westphal H, et al. Cyclopia 738 and defective axial patterning in mice lacking Sonic hedgehog gene function. Nature. 739 1996;383:407-13.

740 28. Eberhart JK, Swartz ME, Crump JG, Kimmel CB. Early Hedgehog signaling from neural to oral epithelium organizes anterior craniofacial development. Development. 2006;133(6):1069-77. Epub 2006/02/17. doi: 10.1242/dev.02281. PubMed PMID: 16481351.

29. Sulik KK, Johnston MC, Webb MA. Fetal Alcohol Syndrome: Embryogenesis in a Mouse Model. Science. 1981;214(4523):936-8.

30. Cohen MM, Sulik KK. Perspectives on holoprosencephaly: Part II. Central nervous system, craniofacial anatomy, syndrome commentary, diagnostic approach, and experimental studies. Journal of craniofacial genetics and developmental biology. 1992;12:196-244.

750 31. Hong M, Krauss RS. Ethanol itself is a holoprosencephaly-inducing teratogen. 751 PLoS One. 2017;12(4):e0176440. Epub 2017/04/26. doi: 10.1371/journal.pone.0176440. 752 PubMed PMID: 28441416; PubMed Central PMCID: PMCPMC5404885. 
32. Kietzman HW, Everson JL, Sulik KK, Lipinski RJ. The teratogenic effects of prenatal ethanol exposure are exacerbated by Sonic Hedgehog or GLI2 haploinsufficiency 755 in the mouse. PLoS One. 2014;9(2):e89448. Epub 2014/03/04. doi: 756 10.1371/journal.pone.0089448. PubMed PMID: 24586787; PubMed Central PMCID: 757 PMCPMC3929747.

758 33. Ahlgren SC, Thakur V, Bronner-Fraser M. Sonic hedgehog rescues cranial neural 759 crest from cell death induced by ethanol exposure. Proc Natl Acad Sci U S A. 760 2002;99(16):10476-81. Epub 2002/07/26. doi: 10.1073/pnas.162356199. PubMed PMID:

761 12140368; PubMed Central PMCID: PMCPMC124946.

762 34. Santos-Ledo A, Cavodeassi F, Carreno H, Aijon J, Arevalo R. Ethanol alters gene 763 expression and cell organization during optic vesicle evagination. Neuroscience. 764 2013;250:493-506. Epub 2013/07/31. doi: 10.1016/j.neuroscience.2013.07.036. PubMed 765 PMID: 23892006; PubMed Central PMCID: PMCPMC3988994.

766 35. Krauss S, Johansen T, Korzh V, Fjose A. Expression of the zebrafish paired box 767 gene pax[zf-b] during early

768 neurogenesis. Development. 1991;113:1193-206.

769 36. Mathers PH, Grinberg A, Mahon KA, Jamrich M. The Rx homeobox gene is 770 essential for vertebrate eye development. Nature. 1997;387:603-7.

771 37. Bénazéraf B, Francois P, Baker RE, Denans N, Little CD, Pourquie O. A random 772 cell motility gradient downstream of FGF controls elongation of an amniote embryo. 773 Nature. 2010;466:248-52.

774 38. Wallingford JB, Fraser SE, Harland RM. Convergent Extension: The Molecular 775 Review Control of Polarized Cell Movement during Embryonic Development. 776 Developmental Cell. 2002;2:695-706.

777 39. Jessen JR, Topczewski J, Bingham S, Sepich DS, Marlow F, Chandrasekhar A, et

778 al. Zebrafish trilobite identifies new roles for Strabismus in gastrulation and neuronal 779 movements. Nat Cell Biol. 2002;4(8):610-5. Epub 2002/07/10. doi: 10.1038/ncb828. 780 PubMed PMID: 12105418; PubMed Central PMCID: PMCPMC2219916.

781 40. Love AM, Prince DJ, Jessen JR. Vang12-dependent regulation of membrane 782 protrusions and directed migration requires a fibronectin extracellular matrix. 783 Development. 2018;145(22). doi: UNSP dev165472

784 10.1242/dev.165472. PubMed PMID: WOS:000451191500007.

785 41. Berres ME, Garic A, Flentke GR, Smith SM. Transcriptome Profiling Identifies 786 Ribosome Biogenesis as a Target of Alcohol Teratogenicity and Vulnerability during Early 787 Embryogenesis. PLoS One. 2017;12(1):e0169351. Epub 2017/01/04. doi: 

PMCPMC5207668.

790 42. Green ML, Singh AV, Zhang Y, Nemeth KA, Sulik KK, Knudsen TB. 791 Reprogramming of genetic networks during initiation of the Fetal Alcohol Syndrome. Dev 792 Dyn. 2007;236(2):613-31. Epub 2007/01/04. doi: 10.1002/dvdy.21048. PubMed PMID: 79317200951.

794 43. Da Lee R, Rhee GS, An SM, Kim SS, Kwack SJ, Seok JH, et al. Differential gene 795 profiles in developing embryo and fetus after in utero exposure to ethanol. J Toxicol 796 Environ Health A. 2004;67(23-24):2073-84. Epub 2004/10/30. doi: 797 10.1080/15287390490515001. PubMed PMID: 15513904.

798 44. Howe K, Clark MD, Torroja CF, Torrance J, Berthelot C, Muffato M, et al. The zebrafish reference genome sequence and its relationship to the human genome. Nature. 800 2013;496(7446):498-503. Epub 2013/04/19. doi: 10.1038/nature12111. PubMed PMID: 23594743 ; PubMed Central PMCID: PMCPMC3703927.

802 45. Marcucio RS, Cordero DR, Hu D, Helms JA. Molecular interactions coordinating 803 the development of the forebrain and face. Dev Biol. 2005;284(1):48-61. Epub 2005/06/28. 804 doi: 10.1016/j.ydbio.2005.04.030. PubMed PMID: 15979605.

805 46. Aoto K, Shikata Y, Imai H, Matsumaru D, Tokunaga T, Shioda S, et al. Mouse Shh 806 is required for prechordal plate maintenance during brain and craniofacial morphogenesis. 807 Dev Biol. 2009;327(1):106-20. Epub 2008/12/24. doi: 10.1016/j.ydbio.2008.11.022. 808 PubMed PMID: 19103193.

809 47. Hong M, Krauss RS. Cdon mutation and fetal ethanol exposure synergize to 810 produce midline signaling defects and holoprosencephaly spectrum disorders in mice. 811 PLoS Genet. 2012;8(10):e1002999. Epub 2012/10/17. doi: 10.1371/journal.pgen.1002999. 812 PubMed PMID: 23071453; PubMed Central PMCID: PMCPMC3469434.

813 48. Chen W, Burgess S, Hopkins N. Analysis of the zebrafish smoothened mutant 814 reveals conserved and divergent functions of hedgehog activity. Development. 2001;128: $815 \quad 2385-96$.

816 49. Brand M, Heisenberg C-P, Warga RM, Pelegri F, Karlstrom RO, Beuchle D, et al. 817 Mutations affecting development of the midline and general body shape during zebrafish 818 embryogenesis. Development. 1996;123: 129-42.

819 50. Yelin R, Schyr RB, Kot H, Zins S, Frumkin A, Pillemer G, et al. Ethanol exposure 820 affects gene expression in the embryonic organizer and reduces retinoic acid levels. Dev 821 Biol. 2005;279(1):193-204. Epub 2005/02/15. doi: 10.1016/j.ydbio.2004.12.014. PubMed 822 PMID: 15708568. 
823 51. Zhang Y, Ding Y, Chen YG, Tao Q. NEDD4L regulates convergent extension 824 movements in Xenopus embryos via Disheveled-mediated non-canonical Wnt signaling. 825 Dev Biol. 2014;392(1):15-25. Epub 2014/05/17. doi: 10.1016/j.ydbio.2014.05.003. 826 PubMed PMID: 24833518.

827 52. Sarmah S, Muralidharan P, Curtis CL, McClintick JN, Buente BB, Holdgrafer DJ, 828 et al. Ethanol exposure disrupts extraembryonic microtubule cytoskeleton and embryonic 829 blastomere cell adhesion, producing epiboly and gastrulation defects. Biol Open. 830 2013;2(10):1013-21. Epub 2013/10/30. doi: 10.1242/bio.20135546. PubMed PMID: 83124167711 ; PubMed Central PMCID: PMCPMC3798184.

832 53. Solnica-Krezel L, Sepich DS. Gastrulation: making and shaping germ layers. Annu 833 Rev Cell Dev Biol. 2012;28:687-717. Epub 2012/07/19. doi: 10.1146/annurev-cellbio834 092910-154043. PubMed PMID: 22804578.

835 54. Keller R. Shaping the Vertebrate Body Plan by

836 Polarized Embryonic Cell Movements. Science. 2002;298:1950-4.

837 55. Solnica-Krezel L. Conserved patterns of cell movements during vertebrate 838 gastrulation. Curr Biol. 2005;15(6):R213-28. Epub 2005/03/31. doi: 839 10.1016/j.cub.2005.03.016. PubMed PMID: 15797016.

840 56. Chuang JC, Raymond PA. Zebrafish genes rx1 and rx2 help define the region of 841 forebrain that gives rise to retina. Dev Biol. 2001;231(1):13-30. Epub 2001/02/22. doi: 842 10.1006/dbio.2000.0125. PubMed PMID: 11180949.

843 57. Roszko I, D SS, Jessen JR, Chandrasekhar A, Solnica-Krezel L. A dynamic 844 intracellular distribution of Vang12 accompanies cell polarization during zebrafish 845 gastrulation. Development. 2015;142(14):2508-20. Epub 2015/06/13. doi: 846 10.1242/dev.119032. PubMed PMID: 26062934; PubMed Central PMCID: 847 PMCPMC4510859.

848 58. Kimmel CB, Ballard WW, Kimmel SR, Ullman B, Schilling TF. Stages of 849 Embryonic Development of the Zebrafish. Developmental Dynamics. 1995;203:253-310.

850 59. Martin M. Cutadapt removes adapter sequences from high-throughput sequencing 851 reads. EMBnetjournal. 2011;17(1):10-2. Epub May 2011.

852 60. Aken BL, Achuthan P, Akanni W, Amode MR, Bernsdorff F, Bhai J, et al. Ensembl 853 2017. Nucleic Acids Res. 2017;45(D1):D635-D42. Epub 2016/12/03. doi: 854 10.1093/nar/gkw1104. PubMed PMID: 27899575; PubMed Central PMCID: 855 PMCPMC5210575. 
856 61. Dobin A, Davis CA, Schlesinger F, Drenkow J, Zaleski C, Jha S, et al. STAR: 857 ultrafast universal RNA-seq aligner. Bioinformatics. 2013;29(1):15-21. Epub 2012/10/30. 858 doi: 10.1093/bioinformatics/bts635. PubMed PMID: 23104886; PubMed Central PMCID: 859 PMCPMC3530905.

860 62. Li H, Handsaker B, Wysoker A, Fennell T, Ruan J, Homer N, et al. The Sequence 861 Alignment/Map format and SAMtools. Bioinformatics. 2009;25(16):2078-9. Epub 862 2009/06/10. doi: 10.1093/bioinformatics/btp352. PubMed PMID: 19505943; PubMed 863 Central PMCID: PMCPMC2723002.

864 63. Anders S, Pyl PT, Huber W. HTSeq--a Python framework to work with high865 throughput sequencing data. Bioinformatics. 2015;31(2):166-9. Epub 2014/09/28. doi: 866 10.1093/bioinformatics/btu638. PubMed PMID: 25260700; PubMed Central PMCID: 867 PMCPMC4287950.

868 64. Love MI, Huber W, Anders S. Moderated estimation of fold change and dispersion 869 for RNA-seq data with DESeq2. Genome Biol. 2014;15(12):550. Epub 2014/12/18. doi: 870 10.1186/s13059-014-0550-8. PubMed PMID: 25516281; PubMed Central PMCID: 871 PMCPMC4302049.

872 65. Leek JT, Storey JD. Capturing Heterogeneity in Gene Expression Studies by 873 "Surrogate Variable Analysis". PLoS Genetics. 2005; preprint(2007). doi: 874 10.1371/journal.pgen.0030161.eor.

875 66. Dixon GB, Davies SW, Aglyamova GV, Meyer E, Bay LK, Matz MV. Genomic 876 determinants of coral heat tolerance across latitudes. Science. 2015;348 (6242).

877 67. Benjamini Y, Hochberg Y. Controlling the False Discovery Rate: A Practical and 878 Powerful Approach to Multiple Testing. Journal of the Royal Statistical Society Series B 879 (Methodological). 1995;57(1):289-300.

880 68. Walker MB, Kimmel CB. A two-color acid-free cartilage and bone stain for 881 zebrafish larvae. Biotech Histochem. 2007;82(1):23-8. Epub 2007/05/19. doi: 882 10.1080/10520290701333558. PubMed PMID: 17510811.

883 69. Miller CT, Schilling TF, Lee K-H, Parker J, Kimmel CB. sucker encodes a 884 zebrafish Endothelin-1 required for ventral pharyngeal arch development. Development. $885 \quad 2000 ; 127: 3815-28$.

886 70. Hothorn T, Bretz F, Westfall P. Simultaneous inference in general parametric 887 models. Biom J. 2008;50(3):346-63. Epub 2008/05/16. doi: 10.1002/bimj.200810425. 888 PubMed PMID: 18481363. 
A

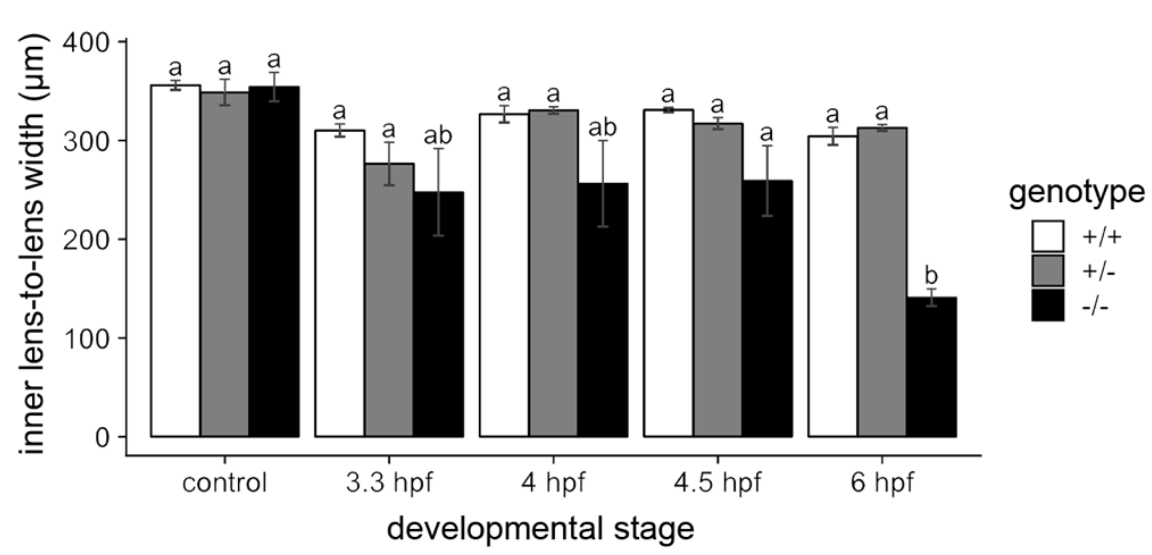

B

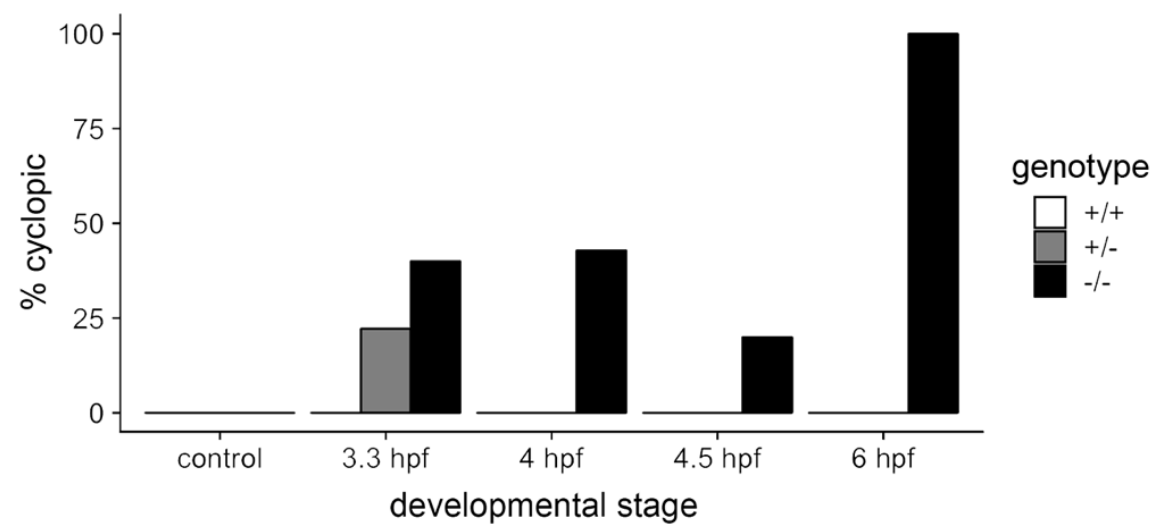

Fig 1. Ethanol interacts with vangl2 during early embryogenesis. (A) Treatment of vangl2 embryos with $1 \%$ ethanol at four different stages comprising late blastula to early gastrula [3.3, 4, 4.5, and 6 hours post fertilization (hpf)] for 24 hours. Homozygous mutants showed a significant decrease in the inner lens-to-lens width when treated from 6 to $30 \mathrm{hpf}$. (B) Heterozygous mutants were most sensitive to ethanol when exposed from 3.3 to 27.3 hpf ( $\mathrm{n}=4 / 18$ cyclopic). Homozygous mutants were most sensitive when exposed from 6 to $30 \mathrm{hpf}(\mathrm{n}=5 / 5$ cyclopic $)$. 


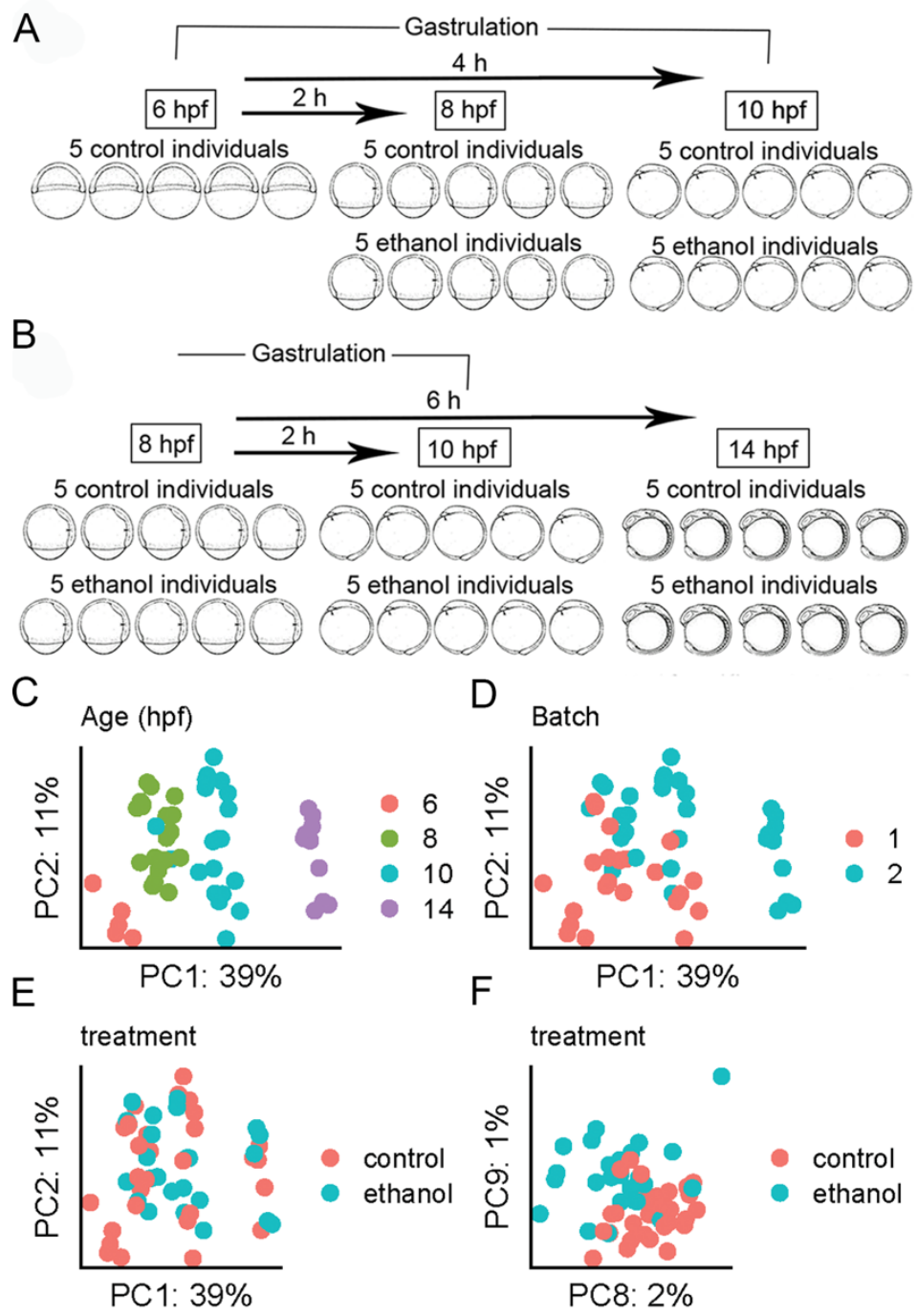

901
Fig 2. Developmental age is the dominant driver of variation in the dataset. (A) Schematic representation of the RNA-seq experimental design. Wild-type AB embryos were exposed to a subteratogenic dose of $1 \%$ ethanol in the embryo media at shield stage (6 hpf). Embryos were subsequently collected at 8 and $10 \mathrm{hpf}$ for experiment 1 and (B) 8, 10 , and $14 \mathrm{hpf}$, for experiment 2 . Each sample consisted of a single zebrafish embryo with 5 control and 5 ethanol-treated samples per timepoint with the exception of the $6 \mathrm{hpf}$ timepoint, which only had 5 control samples. (C-F) Principal components analysis (PCA) of top 25,000 most variable genes. The percentage of variance explained is given on each axis label. (C) $P C 1$ and $P C 2$ color coded by developmental timepoint. (D) $P C 1$ and $P C 2$ color coded by RNA-seq experiment (batch). (E) $P C 1$ and $P C 2$ color coded by ethanol treatment group. (F) $P C 8$ and $P C 9$ showing separation of ethanol treated and control samples. 
A

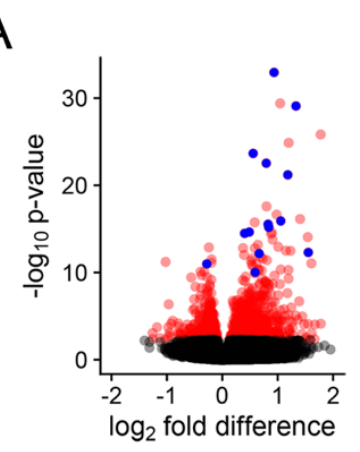

B

Planar cell polarity $(\mathrm{PCP})$ pathway
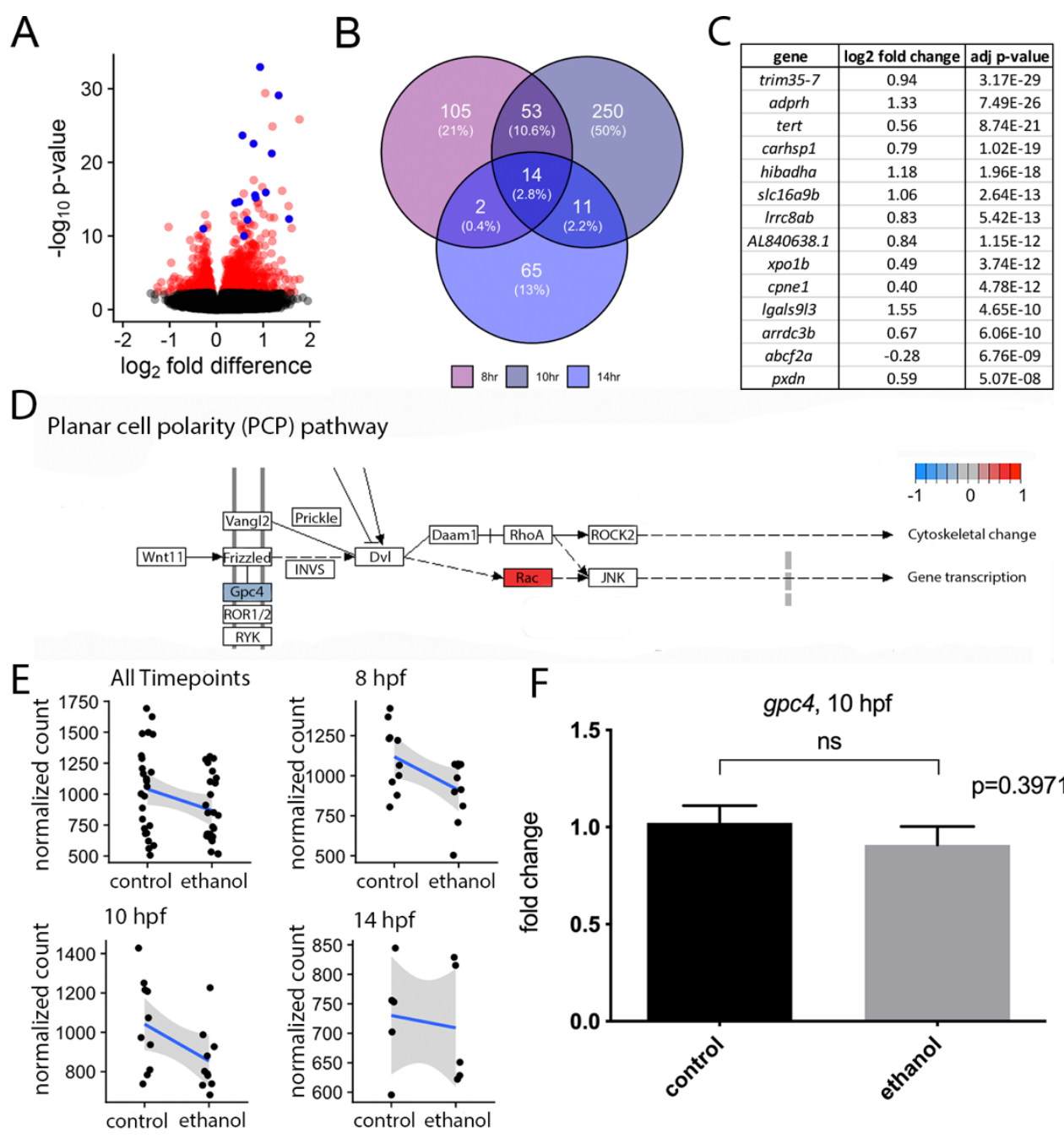

915

916

917

918

919

920

921

922

923

924

925

926

927

928

929

Fig 3. Effects on transcription are largely distinct between developmental time points.

(A) Volcano plot showing differential expression due to ethanol treatment across all samples. Significant genes $(\text { FDR }<0.1)^{*}$ are indicated in red. Genes that were significant at all time points (overlap) are indicated in blue. (B) Venn-diagram showing overlap of significant genes between the three individual timepoints. (C) Table of genes that were significant for each timepoint individually. (D) KEGG pathway schematic illustrating differential expression due to ethanol treatment in the Wnt/planar cell polarity (Wnt/PCP) pathway. Color coding indicates $\log _{2}$ fold differences due to ethanol treatment across all samples, red indicates upregulation due to ethanol, blue indicates downregulation due to ethanol. Only pathway members with significant changes were color coded. In the event that multiple genes from the dataset were annotated with the same pathway member, the $\log _{2}$ fold difference for the gene with the greatest absolute value for the difference was used. 

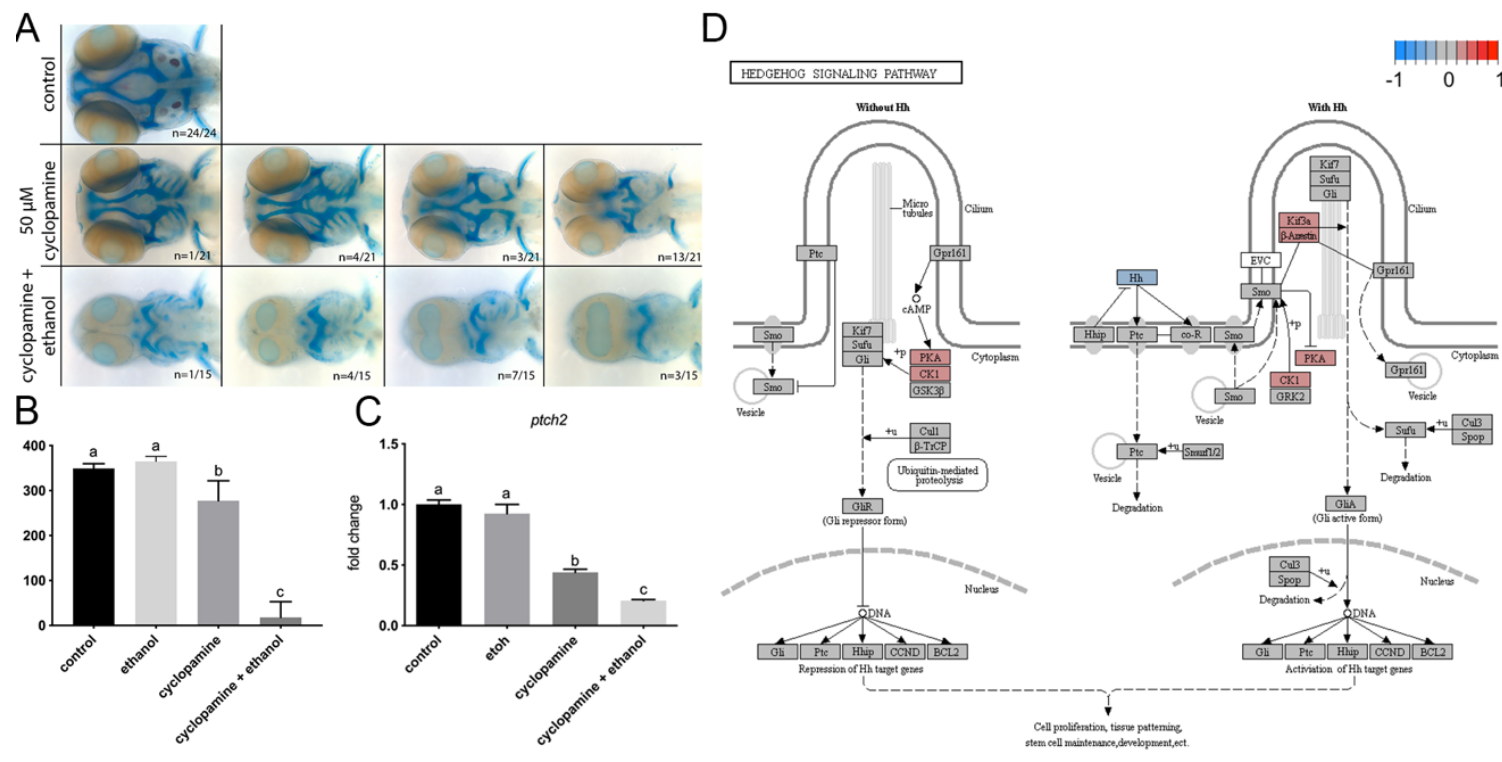

Fig 4. Ethanol indirectly attenuates Shh signaling. (A) Alcian blue and Alizarin red whole mount staining of untreated (control), cyclopamine-treated $(50 \mu \mathrm{M})$, and ethanoland cyclopamine-treated ( $1 \%$ ethanol plus $50 \mu \mathrm{M}$ cyclopamine) wild-type embryos from 6 hpf to $4 \mathrm{dpf}$. Panels represent the spectrum of phenotypes observed for the treatment groups. Embryos were fixed at $4 \mathrm{dpf}$. Dorsal view, anterior to the left. (B) Quantification of the effect of ethanol and cyclopamine on the eye field. Inner lens-to-lens width was used as a morphometric measure of cyclopia. Both cyclopamine alone and cyclopamine and ethanol, were significantly different from controls $(\mathrm{p}<0.0001)$. (C) qRT-PCR of ptch2 in 10 hpf wild-type embryos. slc25a5 (solute carrier family 25) was used as a normalization control. (D) KEGG pathway schematic illustrating differential expression due to ethanol treatment in the sonic hedgehog (Shh) signaling pathway. Color coding indicates $\log _{2}$ fold differences due to ethanol treatment across all samples; red indicates upregulation due to ethanol, blue indicates downregulation due to ethanol. In the event that multiple genes from the dataset were annotated with the same pathway member, the $\log _{2}$ fold difference for the gene with the greatest absolute value for the difference was used. 
A

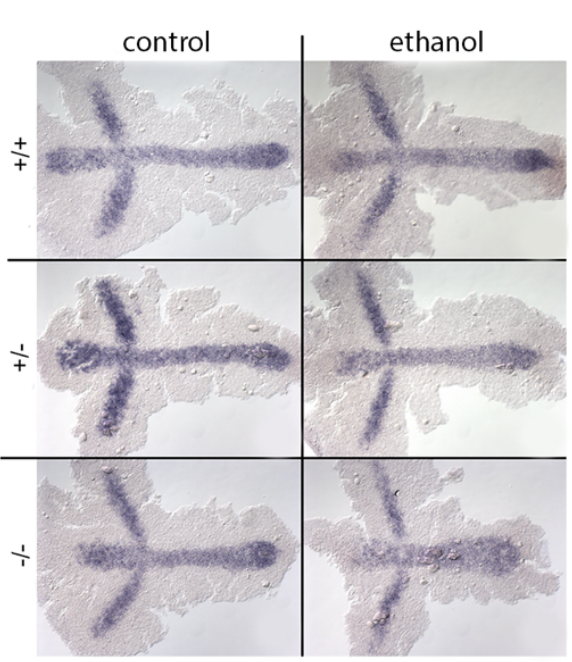

B

C

웡

$$
+/+
$$
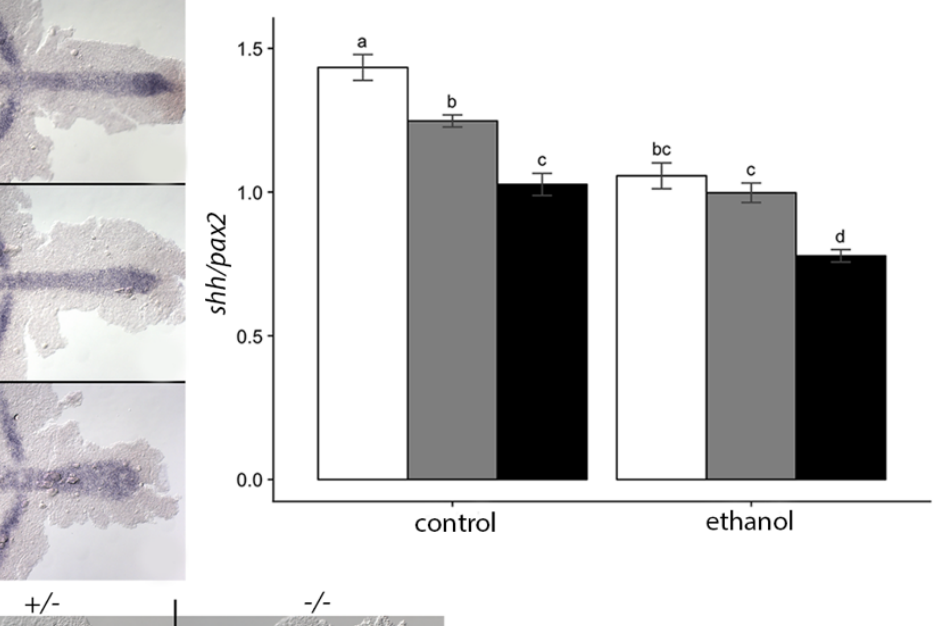

genotype

Fig 5. Ethanol disrupts convergent extension. (A) vangl2 embryos were treated with $1 \%$ ethanol from 3.3 to $10 \mathrm{hpf}$. Embryos were subsequently harvested at $10 \mathrm{hpf}$ for in situ hybridization with shha (midline) and pax2a (midbrain-hindbrain boundary) probes. Dorsal view, anterior to the left. (B) Quantification for the normalized shh/pax2a expression domains were calculated using the AxiovisionLE software. Two-way ANOVA followed by Tukey's post-hoc test was used to analyze the results. Differences were observed between control genotypes and ethanol-treated homozygous mutants. (C) hybridization at $11 \mathrm{hpf}$. 
A

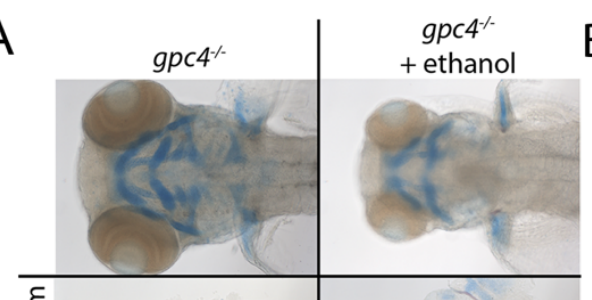

B

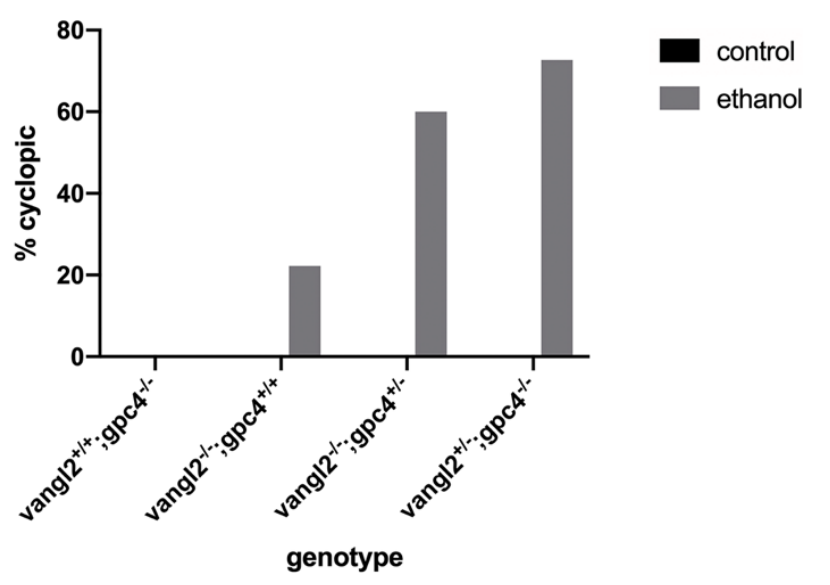

Fig 6. Mutation in gpc4 exacerbates cyclopia in a dose-dependent manner. (A)

960 Normalized read counts indicating expression of gpc4 across different subsets of the 961 dataset. (B) qRT-PCR of gpc4 in 10 hpf wild-type embryos. actbl (actin, beta 1), lsm 12b 962 (like-Sm protein 12 homolog b), and slc25a5 (solute carrier family 25) were used for 963 normalization. (C) Alcian blue and Alizarin red whole- and flat-mount staining of untreated 964 and 1\% ethanol-treated (6 hpf - $4 \mathrm{dpf}$ ) gpc4 homozygous mutants. Embryos fixed at $4 \mathrm{dpf}$. 965 Dorsal view, anterior to the left. (D) Functional analyses of vangl2; gpc4 double mutants. 966 Enhanced cyclopia was observed in ethanol-treated vangl2;gpc4 double mutants with loss 967 of at least one copy of either gene. 


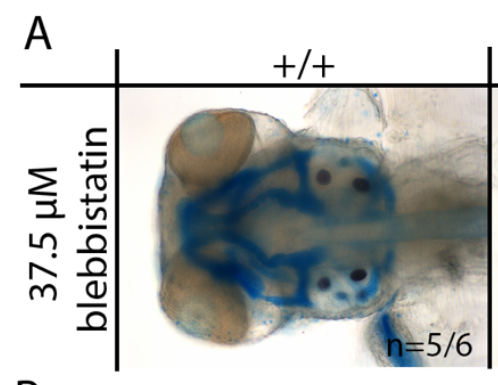

\section{B}

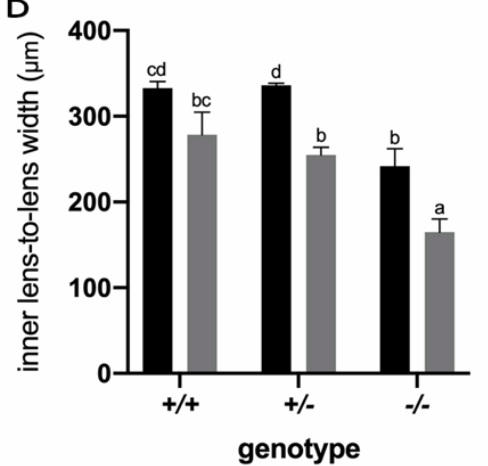

C
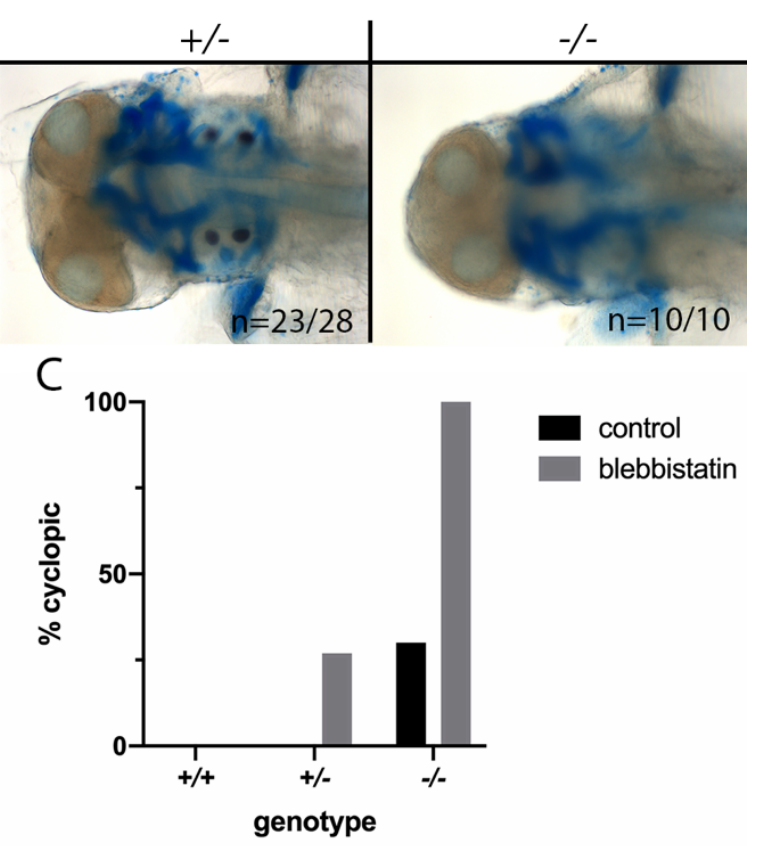

Fig 7. Blebbistatin phenocopies ethanol treatment in vangl2 mutant embryos. (A)

972 Alcian blue and Alizarin red whole-mount staining of vangl2 embryos treated with $37.5 \mu \mathrm{M}$

973 blebbistatin (6-10 hpf). Embryos fixed at $4 \mathrm{dpf}$. Dorsal view, anterior to the left. (B)

974 Quantification of the inner lens-to-lens width of control and blebbistatin-treated embryos.

975 Lettering indicates differences in significance based on multiple comparisons test using

976 Tukey's HSD. (C) Representation of the percentage of control and blebbistatin-treated

977 embryos exhibiting cyclopia. Genotypes indicated on the x-axes. 

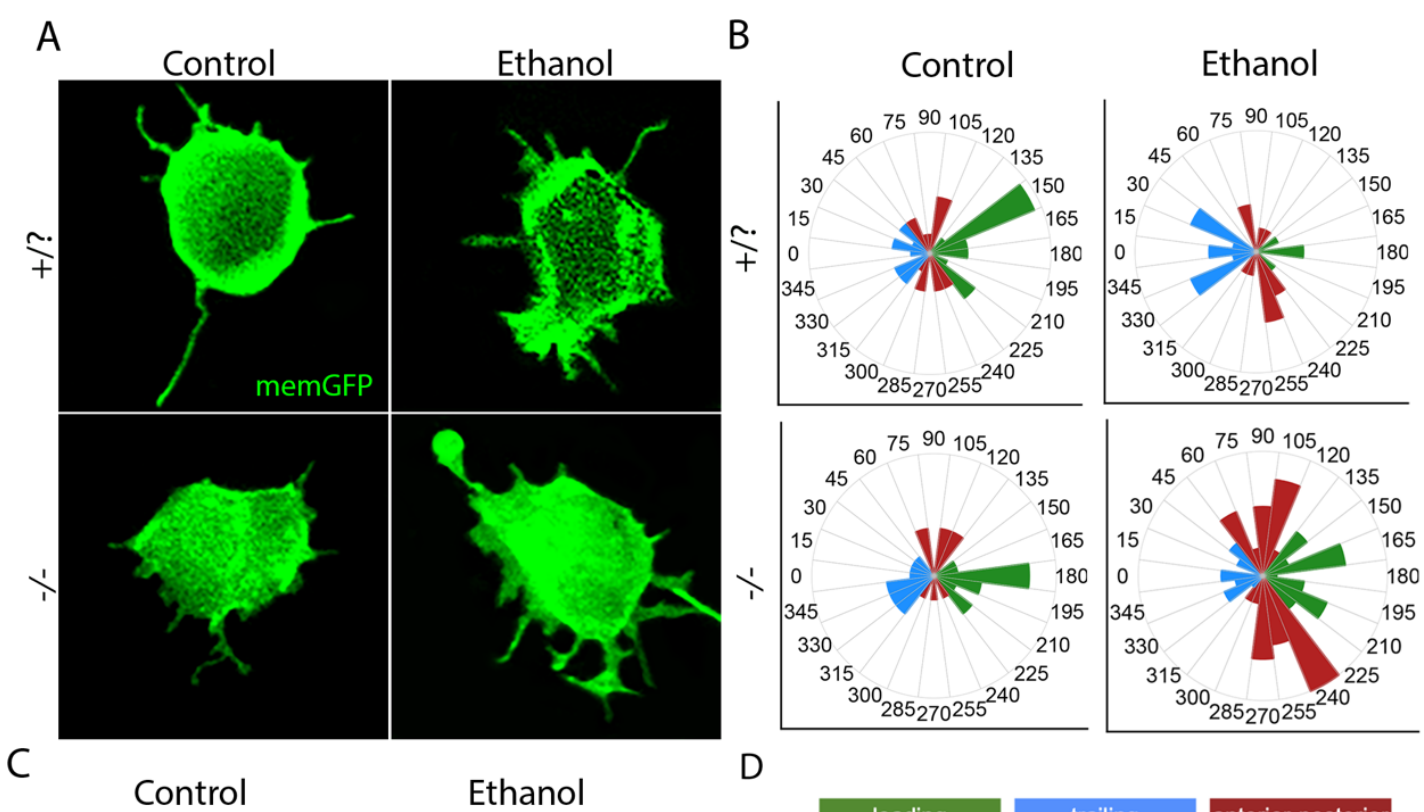

\section{Ethanol}
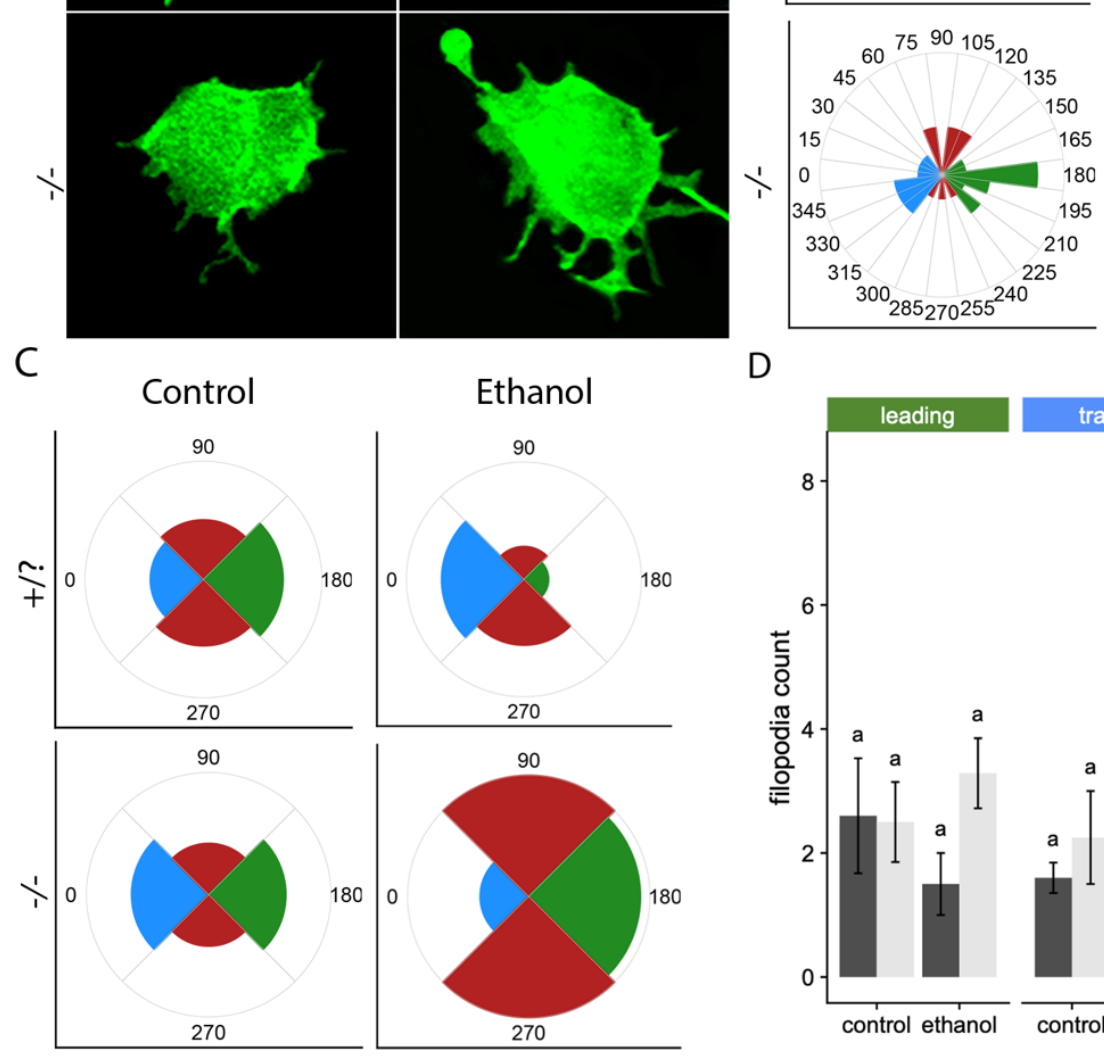

Fig 8. Membrane protrusions in untreated and ethanol-treated (6-10 hpf) vangl2 embryos. (A) Confocal images of untreated and ethanol-treated vangl 2 mesodermal cells expressing memGFP at $10 \mathrm{hpf}$. $\mathrm{n}=6$ (B) Rose diagram of filopodial projections representing length and distribution around the circumference of a cell. 24 bins, divided into $15^{\circ}$ angles (C) Rose diagram of filopodial projections representing length and distribution around the circumference of a cell. 4 bins, divided into $90^{\circ}$ angles. Horizontal plane $\left(0^{\circ}\right)$ represents the

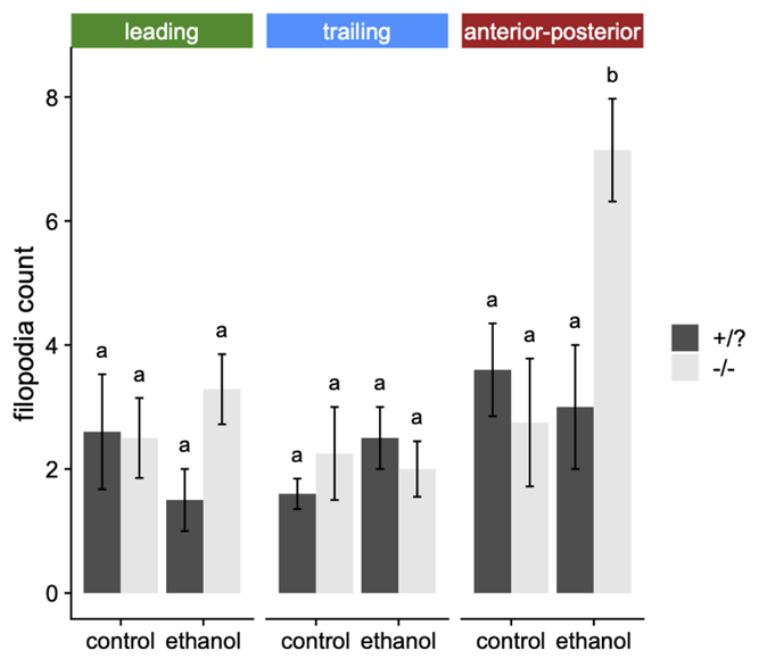
mediolateral axis; vertical plane $\left(90^{\circ}\right)$ represents the anterior-posterior axis. 
bioRxiv preprint doi: https://doi.org/10.1101/805556; this version posted June 18, 2020. The copyright holder for this preprint (which was not certified by peer review) is the author/funder. All rights reserved. No reuse allowed without permission.

992

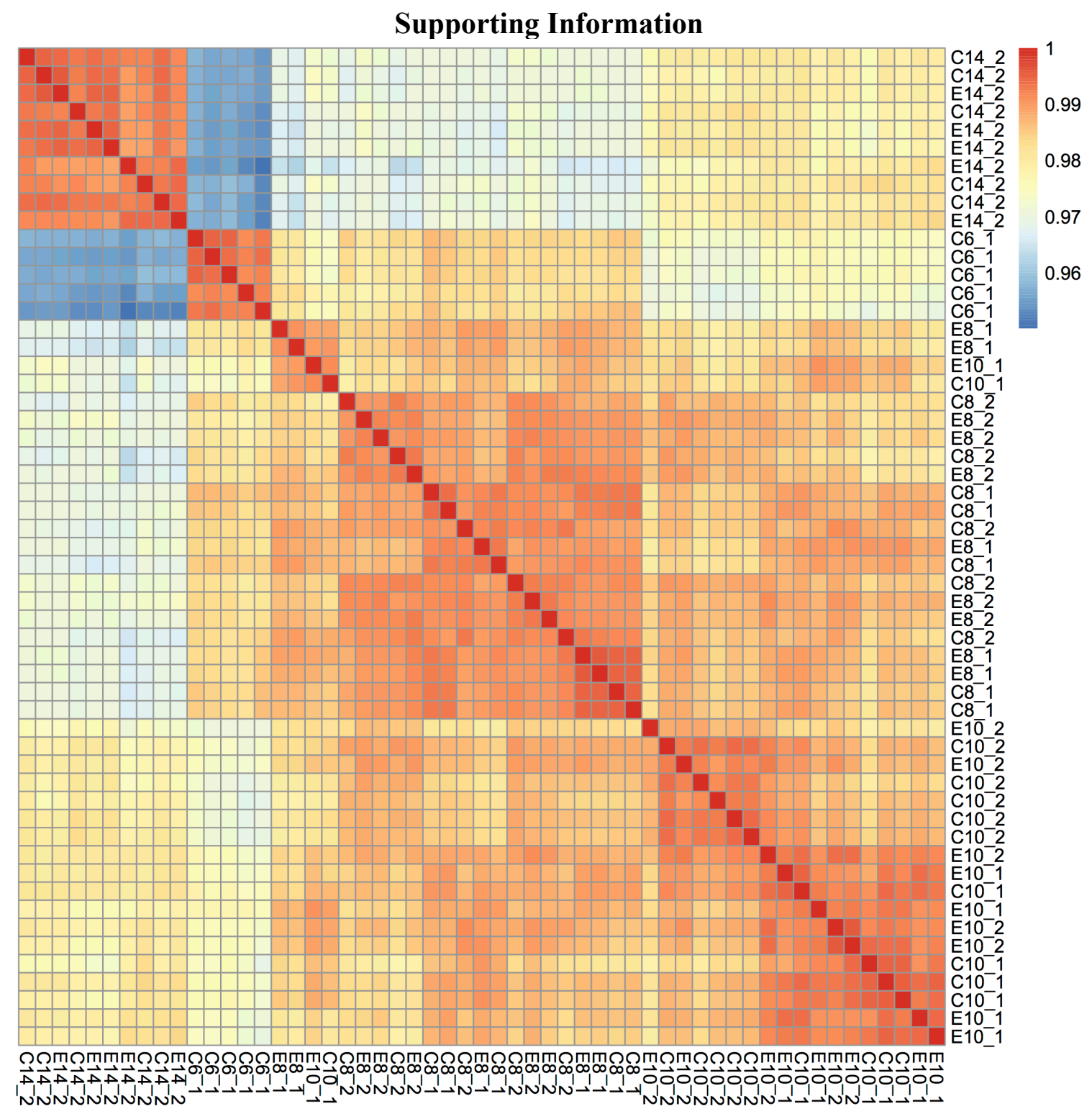

993

994

995

996

997

998
S1 Fig. Time is the major driver of variation in the dataset. Heatmap showing overall correlation of gene expression among samples. Samples were hierarchically clustered based on similarity. Treatment [control or ethanol treated $(\mathrm{C}$ or $\mathrm{E})$ ] and developmental age [hours post fertilization $(6,10,8$, or 14$)]$ are indicated in the row and column labels. 


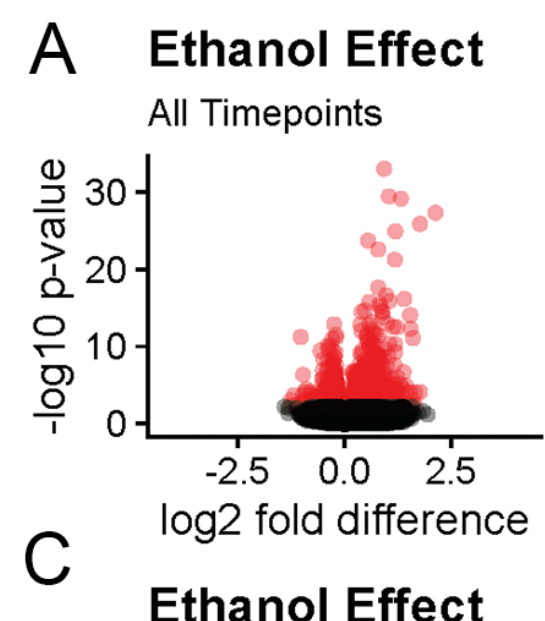

10 hour

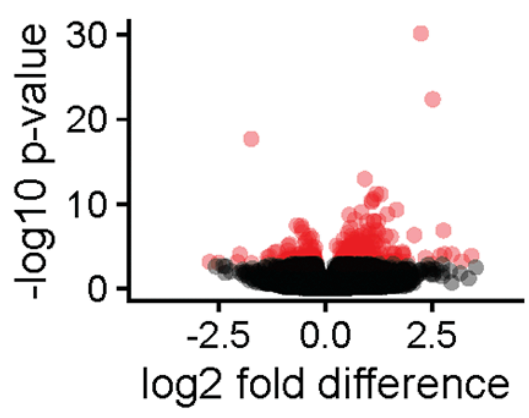

\section{B Ethanol Effect}

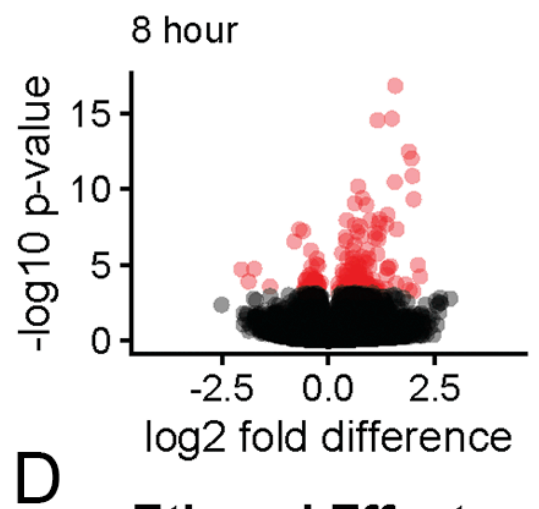

\section{Ethanol Effect}

14 hour

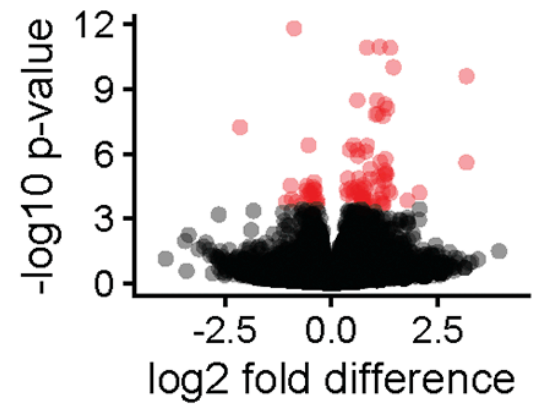

999

1000

1001

1002

1003

1004

1005

1006

S2 Fig. There are more upregulated than downregulated genes among ethanoltreated individuals. Volcano plot showing variation in the transcriptional response to ethanol treatment across developmental timepoints. Significant genes (FDR $<0.1$ ) are indicated in red. For each subset, the names of the topmost significantly dysregulated genes are noted near gene's data point (A) All timepoints combined together (B) 8 hpf only (C) 10 hpf only (D) 14 hpf only. 

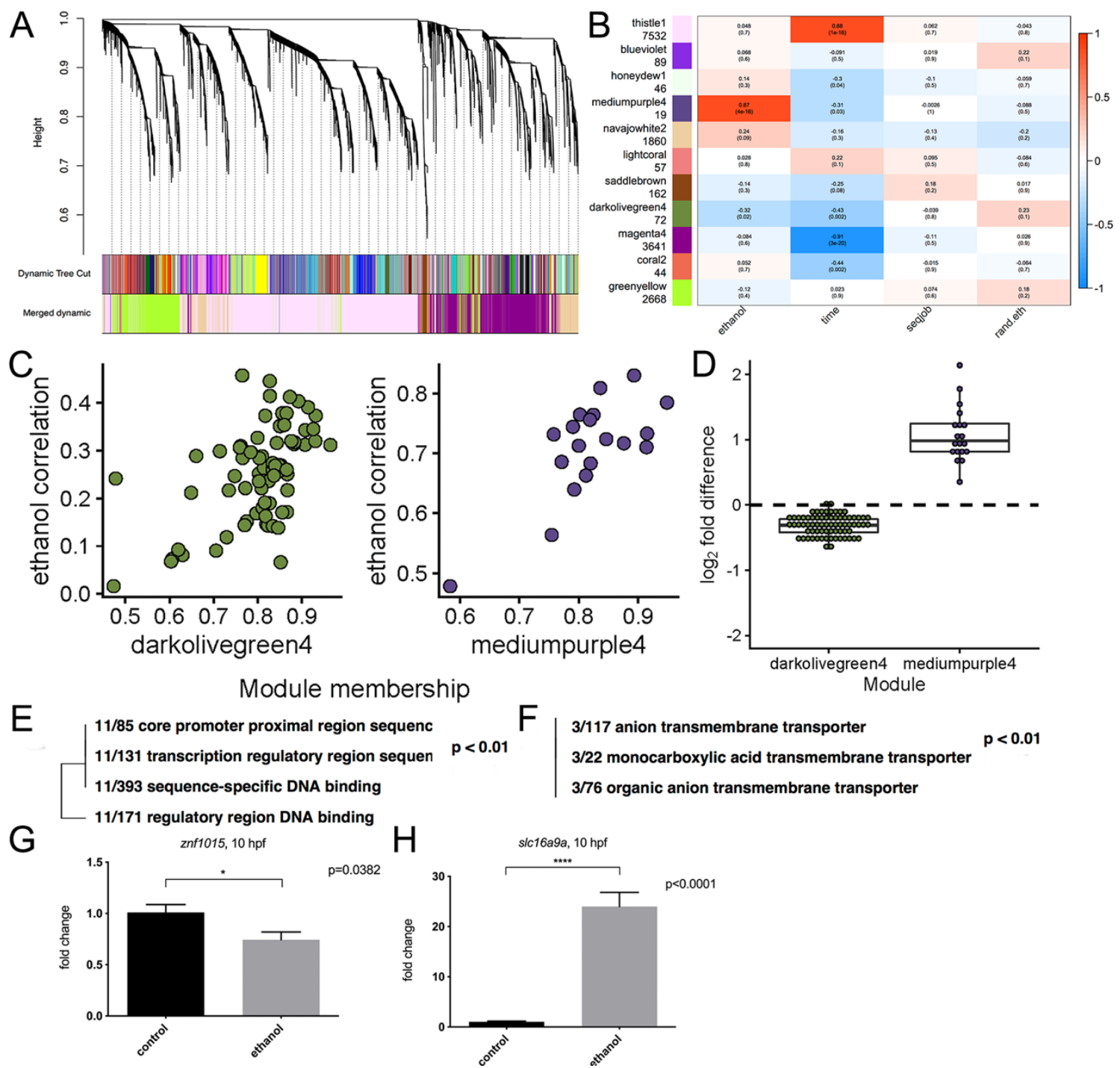

1007
S3 Fig. WGCNA identifies two modules that are significantly correlated with ethanol exposure. (A) Dendrogram illustrating the hierarchical clustering of the genes and with their corresponding modules colors. The top layer of colors indicates the Dynamic Tree Cutoff, including all assigned modules, before merging by module similarity. The bottom layer indicates the module colors after merging. These merged modules were used for further analysis. (B) Heatmap of module-trait correlations. The eigengene for each module was correlated with ethanol treatment (ethanol), hours post fertilization (age), experimental batch (seqjob), and as a negative control, a randomly shuffled version of the ethanol treatments (rand.eth). Intensity of the color in each cell indicates the strength of correlation between the module (row labels) and the sample trait (column labels). Two modules, (mediumpurple4 and darkolivegreen4) significantly correlated with ethanol treatment $(\mathrm{p}<$ 0.05). (C) Scatterplots of correlation with ethanol treatment against module membership. Each datapoint is a gene assigned to the indicated module. Ethanol correlation is the Pearson correlation between the gene's expression level and ethanol treatment. Module 
1022 membership is the correlation between the genes expression level and the module 1023 eigengene and describes how well the gene matches the overall patterns of the module. (D) 1024 Boxplot of $\log _{2}$ fold differences due to ethanol for the two significant modules. (E) Gene 1025 ontology enrichment tree for Molecular Function for the darkolivegreen4 module. (F) Gene 1026 ontology enrichment tree for Molecular Function for the mediumpurple4 module. (G) 1027 Changes in gene expression from the RNA-seq were validated using wild-type embryos at 102810 hpf. slc16a9a was selected from the mediumpurple4 module and $(\mathrm{H})$ znflo15 was 1029 selected from the darkolivegreen 4 module. Fold change indicates the degree of change 1030 between untreated control and ethanol-treated stage-matched embryos. lsm 12b (like-Sm 1031 protein 12 homolog $b$ ) was used as a normalization control gene. 

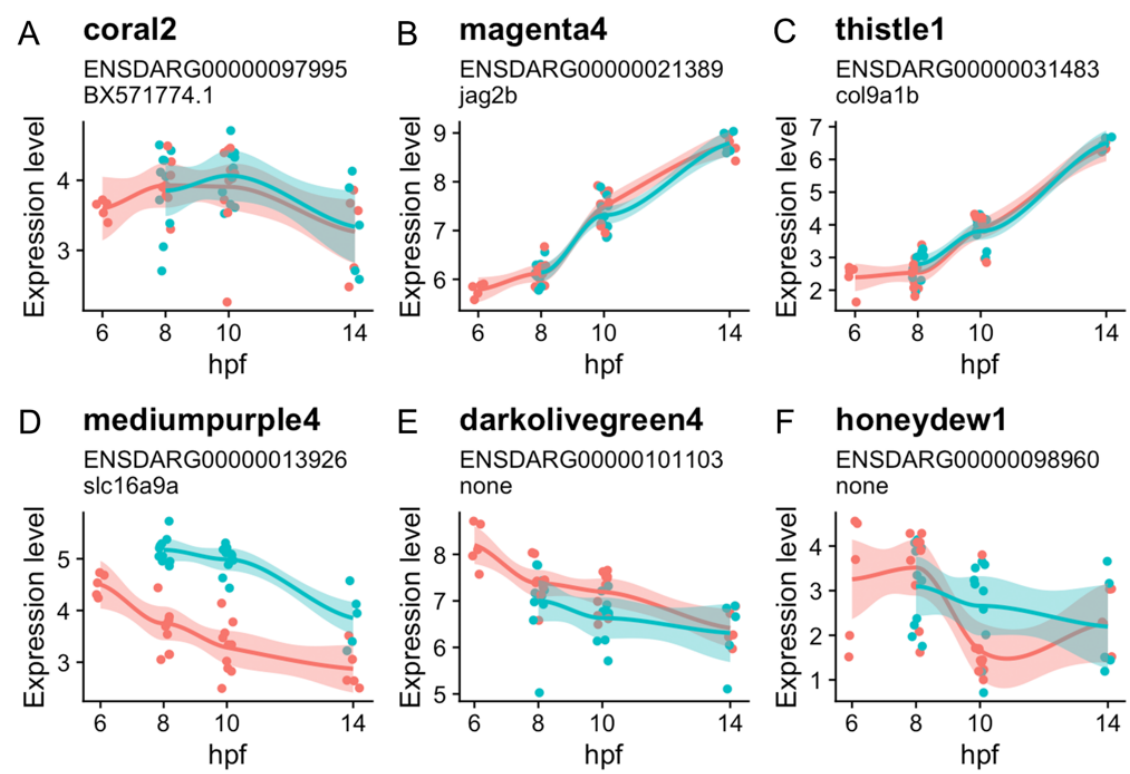

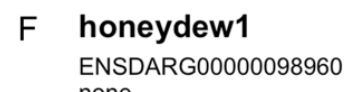
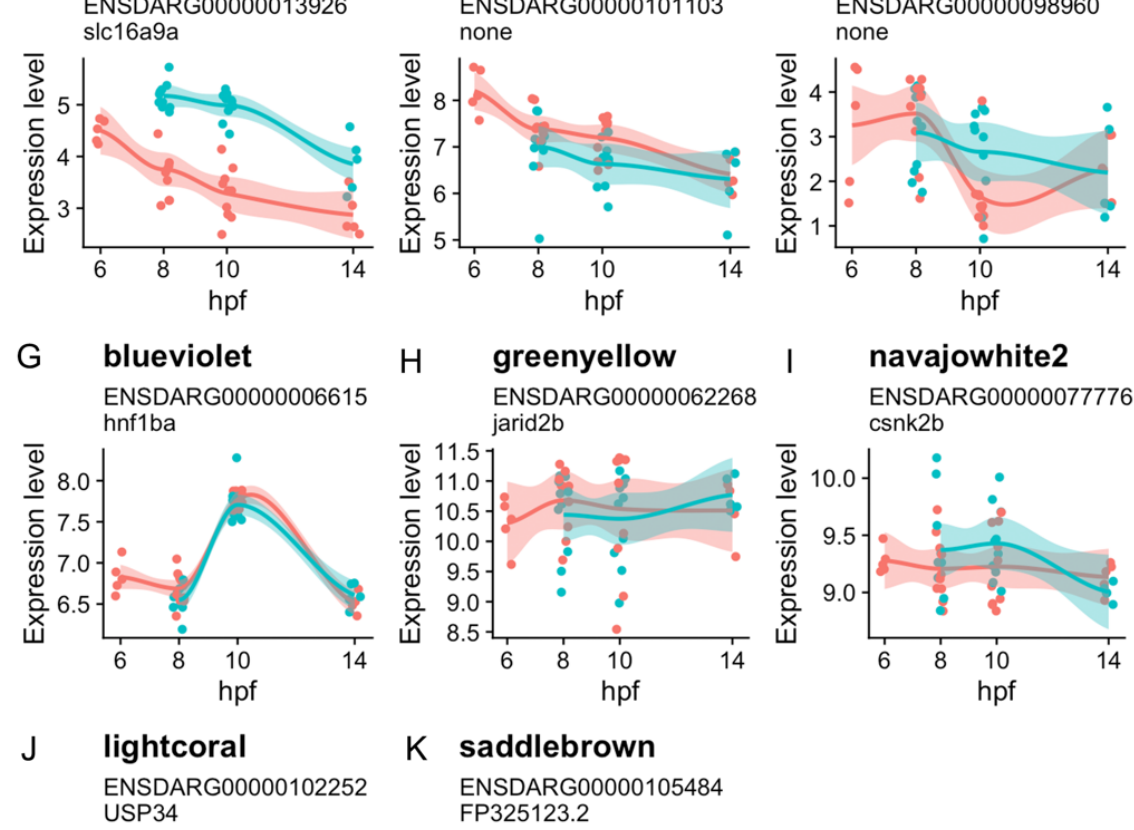

$\mathrm{K}$ saddlebrown

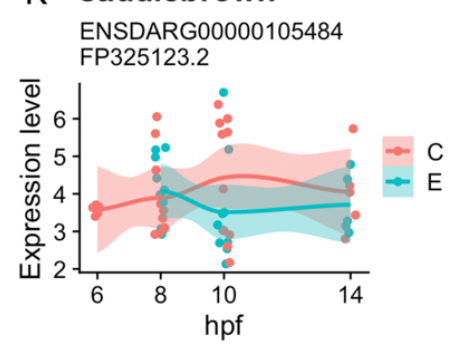

\section{S4 Fig. Expression of hub genes for development-related modules indicate ethanol did} not retard developmental progression. (A-K) Development-related WGCNA modules were identified as those with significant relationship to hours post fertilization (Pearson correlation; $\mathrm{p}<0.05$ ). The hub gene for each of these modules was identified as the gene assigned to that module with the highest module membership (defined as the correlation of the gene's expression level with the module eigengene). Normalized expression levels for the hub genes were plotted against hours post fertilization. The relationship between expression and time is largely consistent between the ethanol treated (E, teal) and control (C, pink) samples. Even for the two modules associated with ethanol treatment (mediumpurple4 and darkolivegreen4), the slopes of the lines are very similar, indicating subteratogenic ethanol exposure did not cause significant developmental delay. 

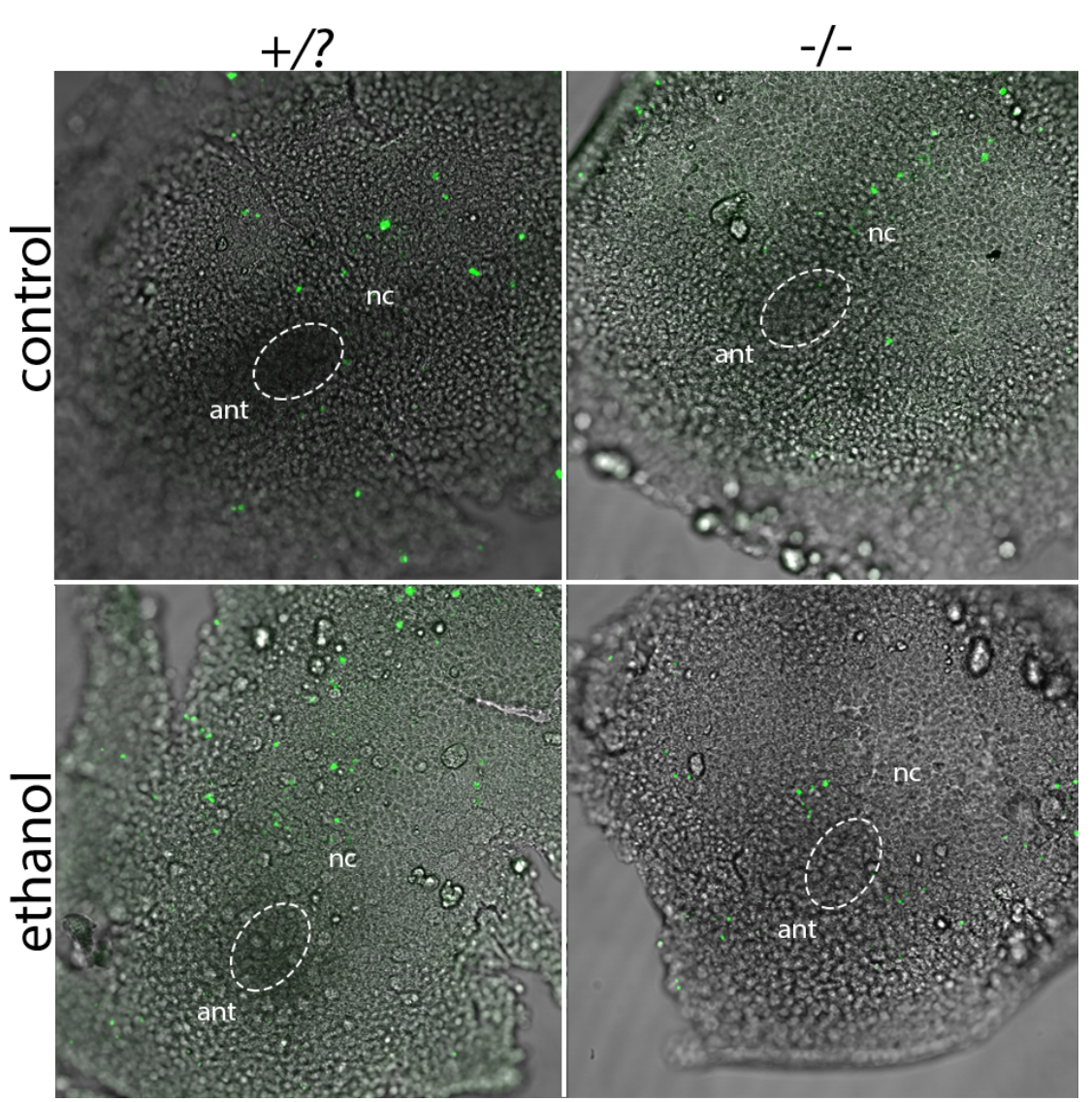

1048

S5 Fig. Cell death by TUNEL at 11 hpf in untreated and ethanol-treated vangl2

1049 mutants. The number of positive cells in the eye field (indicated by dashed circle) was not 1050 higher in homozygous or ethanol-treated mutants. ant $=$ anterior; $\mathrm{nc}=$ notochord 


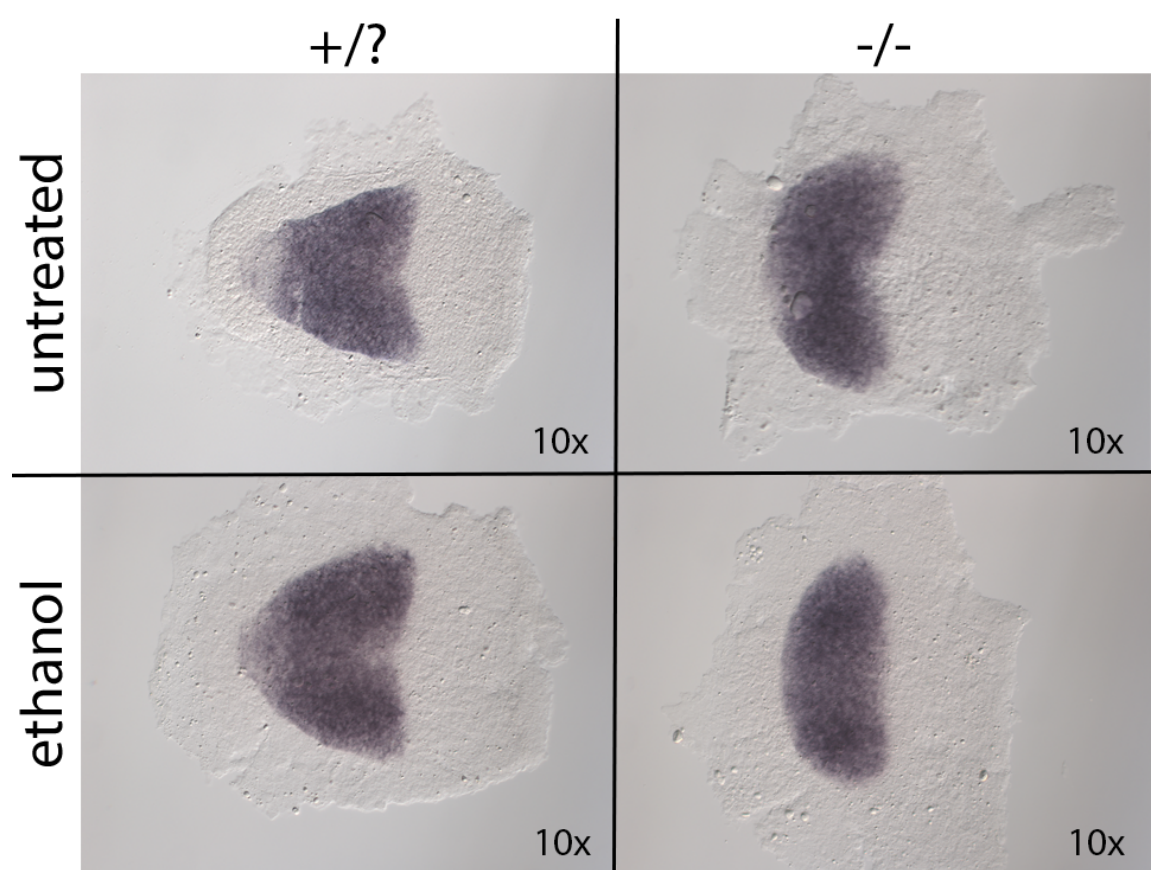

1052 S6 Fig. Ethanol alters $r \boldsymbol{x} 3$ expression in the eye field. Expression pattern of transcription 1053 factor $r x 3$, stained using whole mount in situ hybridization at $12 \mathrm{hpf}$. Dorsal view, anterior 1054 to the left.

1055 


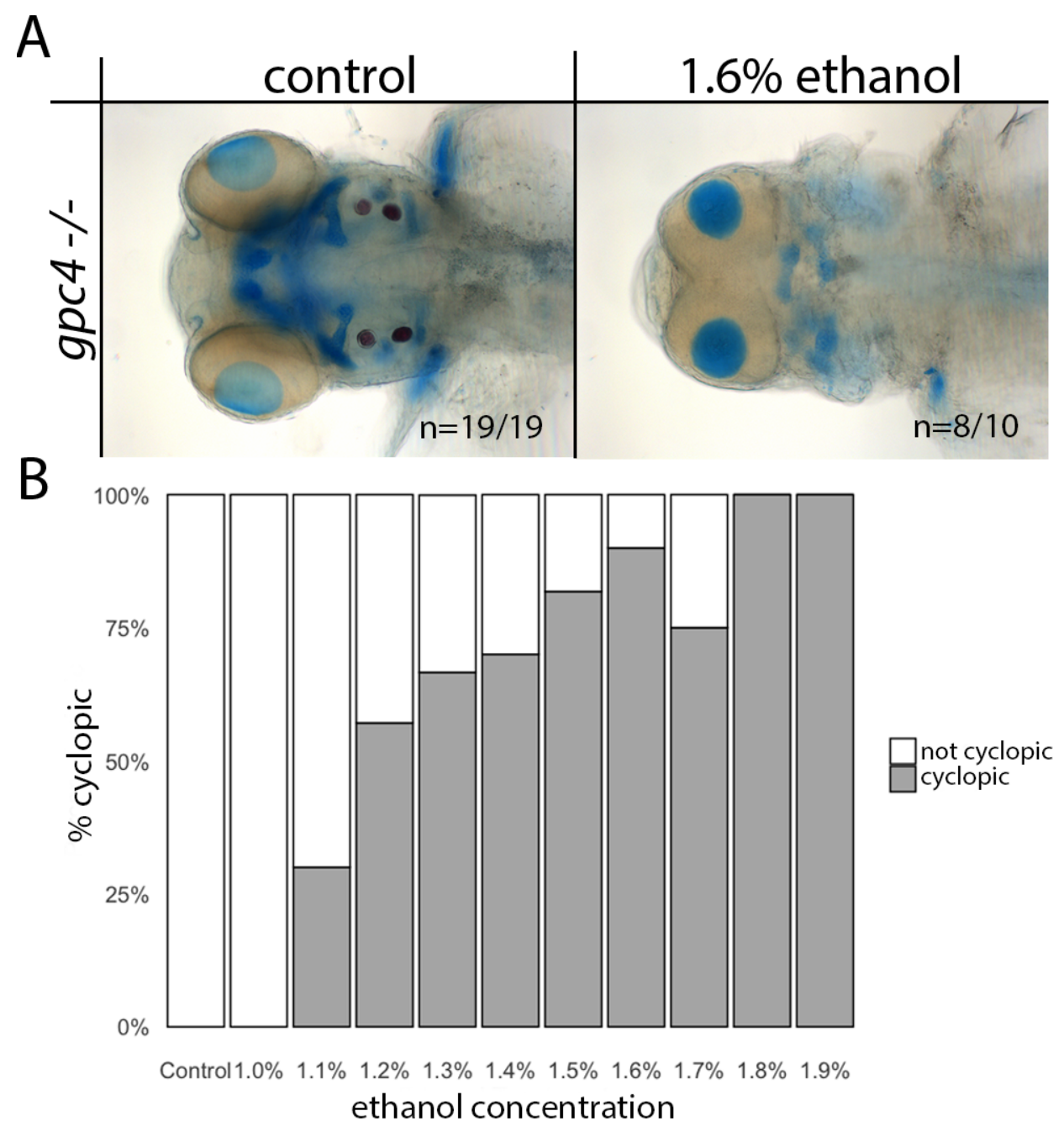

1056

S7 Fig. Ethanol interacts with gpc4. (A) Alcian blue and Alizarin red whole-mount staining of untreated and $1.6 \%$ ethanol-treated ( $6 \mathrm{hpf}-24 \mathrm{hpf})$ gpc 4 homozygous mutants. Embryos fixed at $4 \mathrm{dpf}$. Dorsal view, anterior to the left. (B) Dose-response curve of gpc4 mutants treated with $1-1.9 \%$ ethanol (6-30 hpf). 